Proceedings of the Edinburgh Mathematical Society (2004) 47, 163-190 (C)

DOI:10.1017/S0013091502000718 Printed in the United Kingdom

\title{
GENERATORS FOR $\mathcal{H}$-INVARIANT PRIME IDEALS IN $O_{q}\left(\mathcal{M}_{m, p}(\mathbb{C})\right)$
}

\author{
STÉPHANE LAUNOIS \\ Laboratoire de Mathématiques, UMR 6056, Université de Reims, Moulin de la Housse, \\ BP 1039-51687 REIMS Cedex 2, France (stephane.launois@univ-reims.fr)
}

(Received 22 July 2002)

\begin{abstract}
It is known that, for generic $q$, the $\mathcal{H}$-invariant prime ideals in $O_{q}\left(\mathcal{M}_{m, p}(\mathbb{C})\right)$ are generated by quantum minors (see S. Launois, Les idéaux premiers invariants de $O_{q}\left(\mathcal{M}_{m, p}(\mathbb{C})\right)$, J. Alg., in press). In this paper, $m$ and $p$ being given, we construct an algorithm which computes a generating set of quantum minors for each $\mathcal{H}$-invariant prime ideal in $O_{q}\left(\mathcal{M}_{m, p}(\mathbb{C})\right)$. We also describe, in the general case, an explicit generating set of quantum minors for some particular $\mathcal{H}$-invariant prime ideals in $O_{q}\left(\mathcal{M}_{m, p}(\mathbb{C})\right)$. In particular, if $\left(Y_{i, \alpha}\right)_{(i, \alpha) \in[1, m] \times \llbracket 1, p]}$ denotes the matrix of the canonical generators of $O_{q}\left(\mathcal{M}_{m, p}(\mathbb{C})\right)$, we prove that, if $u \geqslant 3$, the ideal in $O_{q}\left(\mathcal{M}_{m, p}(\mathbb{C})\right)$ generated by $Y_{1, p}$ and the $u \times u$ quantum minors is prime. This result allows Lenagan and Rigal to show that the quantum determinantal factor rings of $O_{q}\left(\mathcal{M}_{m, p}(\mathbb{C})\right)$ are maximal orders (see T. H. Lenagan and L. Rigal, Proc. Edinb. Math. Soc. 46 (2003), 513-529).
\end{abstract}

Keywords: quantum matrices; quantum minors; prime ideals; quantum determinantal ideals; deleting-derivations algorithms

2000 Mathematics subject classification: Primary 16P40

Secondary 16W35; 20G42

\section{Introduction}

Fix two positive integers $m$ and $p$ with $m, p \geqslant 2$ and consider some complex number $q$ which is transcendental over $\mathbb{Q}$. Denote by $R=O_{q}\left(\mathcal{M}_{m, p}(\mathbb{C})\right)$ the quantization of the ring of regular functions on $m \times p$ matrices with entries in $\mathbb{C}$ (the field of complex numbers) and let $\left(Y_{i, \alpha}\right)_{(i, \alpha) \in \llbracket 1, m \rrbracket \times \llbracket 1, p \rrbracket}$ denote the matrix of its canonical generators. There is an action of the torus $\mathcal{H}=\left(\mathbb{C}^{*}\right)^{m+p}$ on $R$ by $\mathbb{C}$-automorphisms via

$$
\left(a_{1}, \ldots, a_{m}, b_{1}, \ldots, b_{p}\right) \cdot Y_{i, \alpha}=a_{i} b_{\alpha} Y_{i, \alpha} \quad((i, \alpha) \in \llbracket 1, m \rrbracket \times \llbracket 1, p \rrbracket) .
$$

(If $m=p$, this action is induced by the bialgebra structure of $R$ and, if $m \neq p$, it is easy to check that the relations which define $R$ are preserved by the group $\mathcal{H}$.)

It is known from work of Goodearl and Letzter that $R$ has only finitely many $\mathcal{H}$ invariant prime ideals (see $[\mathbf{8}]$ ) and that, in order to calculate the prime and primitive spectra of $R$, it is enough to determine the $\mathcal{H}$-invariant prime ideals of $R$ (see [8, Theorem 6.6]).

In [10], we proved that the $\mathcal{H}$-invariant prime ideals in $R$ are generated by quantum minors, as conjectured by Goodearl and Lenagan (see $[\mathbf{5}]$ and $[\mathbf{6}]$ ). In this paper, we use 
this result, together with Cauchon's description for the set of $\mathcal{H}$-invariant prime ideals of $R$ (see [3, Théorème 3.2.1]), to construct an algorithm which provides an explicit generating set of quantum minors for each $\mathcal{H}$-invariant prime ideal in $R$ (see $\S 4$ ). (Of course, these generating sets can be computed with this algorithm only when $m$ and $p$ have fixed values.)

The last part of this paper is devoted to the general case. We construct certain sets of quantum minors which generate prime ideals of $R$. In order to do that, we consider a new deleting-derivations algorithm (see $[\mathbf{2}]$ ) that we define in $\S 5$. Using this new tool, we can prove that, if $u \geqslant 3$, the ideal in $O_{q}\left(\mathcal{M}_{m, p}(\mathbb{C})\right)$ generated by $Y_{1, p}$ and the $u \times u$ quantum minors is prime. This result allows Lenagan and Rigal [11] to show that the quantum determinantal factor rings of $O_{q}\left(\mathcal{M}_{m, p}(\mathbb{C})\right)$ are maximal orders.

\section{2. $\mathcal{H}$-invariant prime ideals in $O_{q}\left(\mathcal{M}_{m, p}(\mathbb{C})\right)$}

Throughout this paper, we use the following conventions.

(i) $\mathbb{N}, \mathbb{Q}$ and $\mathbb{C}$ denote, respectively, the set of natural numbers, the field of rational numbers and the field of complex numbers. We set $\mathbb{C}^{*}=\mathbb{C} \backslash\{0\}$.

(ii) If $I$ is any non-empty finite subset of $\mathbb{N},|I|$ denotes its cardinality.

(iii) $q \in \mathbb{C}$ is transcendental over $\mathbb{Q}$.

(iv) $m$ and $p$ denote two positive integers with $m, p \geqslant 2$.

(v) If $k$ is a positive integer, $S_{k}$ denotes the group of permutations of $\llbracket 1, k \rrbracket$.

(vi) $R=O_{q}\left(\mathcal{M}_{m, p}(\mathbb{C})\right)$ denotes the quantization of the ring of regular functions on $m \times p$ matrices with entries in $\mathbb{C}$; it is the $\mathbb{C}$-algebra generated by the $m \times p$ indeterminates $Y_{i, \alpha}, 1 \leqslant i \leqslant m$ and $1 \leqslant \alpha \leqslant p$, subject to the following relations.

If

$$
\left(\begin{array}{ll}
x & y \\
z & t
\end{array}\right)
$$

is any $2 \times 2$ sub-matrix of $\mathcal{Y}=\left(Y_{i, \alpha}\right)_{(i, \alpha) \in \llbracket 1, m \rrbracket \times \llbracket 1, p \rrbracket}$, then

(a) $y x=q^{-1} x y, z x=q^{-1} x z, z y=y z, t y=q^{-1} y t, t z=q^{-1} z t$;

(b) $t x=x t-\left(q-q^{-1}\right) y z$.

These relations agree with the relations used in $[\mathbf{3}],[\mathbf{5}],[\mathbf{6}],[\mathbf{1 0}]$ and $[\mathbf{1 1}]$, but they differ from those of $[\mathbf{1 2}]$ by an interchange of $q$ and $q^{-1}$. It is well known that the ring $R$ is a Noetherian domain. We denote by $F$ its skew field of fractions. Moreover, since $q$ is transcendental over $\mathbb{Q}$, it follows from [7, Theorem 2.3] that all prime ideals of $R$ are completely prime. 
(vii) As in $[3, \S 2.1]$, one can show that the group $\mathcal{H}=\left(\mathbb{C}^{*}\right)^{m+p}$ acts on $R$ by $\mathbb{C}$-algebra automorphisms via

$$
\left(a_{1}, \ldots, a_{m}, b_{1}, \ldots, b_{p}\right) . Y_{i, \alpha}=a_{i} b_{\alpha} Y_{i, \alpha} \quad \forall(i, \alpha) \in \llbracket 1, m \rrbracket \times \llbracket 1, p \rrbracket .
$$

(viii) An $\mathcal{H}$-eigenvector $x$ of $R$ is a non-zero element $x \in R$ such that $h . x \in \mathbb{C}^{*} x$ for each $h \in \mathcal{H}$. An ideal $I$ of $R$ is said to be $\mathcal{H}$-invariant if $h . I=I$ for all $h \in \mathcal{H}$. Let $\mathcal{H}$-Spec $(R)$ denote the set of $\mathcal{H}$-invariant prime ideals of $R$.

The aim of this section is to describe the set $\mathcal{H}-\operatorname{Spec}(R)$ by using the standard deletingderivations algorithm (see $[\mathbf{1 0}, \S 2.1])$.

\section{Notation 2.1.}

(i) We denote by $\leqslant_{\mathrm{s}}$ the lexicographic ordering on $\mathbb{N}^{2}$. We often call it the standard ordering on $\mathbb{N}^{2}$. Recall that

$$
(i, \alpha) \leqslant_{\mathrm{s}}(j, \beta) \Longleftrightarrow[(i<j) \text { or }(i=j \text { and } \alpha \leqslant \beta)] .
$$

(ii) We set $E_{\mathrm{s}}=(\llbracket 1, m \rrbracket \times \llbracket 1, p \rrbracket \cup\{(m, p+1)\}) \backslash\{(1,1)\}$.

(iii) Let $(j, \beta) \in E_{\mathrm{s}}$. If $(j, \beta) \neq(m, p+1),(j, \beta)^{+_{\mathrm{s}}}$ denotes the smallest element (relative to $\left.\leqslant_{\mathrm{s}}\right)$ of the set $\left\{(i, \alpha) \in E_{\mathrm{s}} \mid(j, \beta)<_{\mathrm{s}}(i, \alpha)\right\}$.

Notation 2.2. If $r=(j, \beta)$ and $v=(i, \alpha)$ belong to $\llbracket 1, m \rrbracket \times \llbracket 1, p \rrbracket$, we define a complex number $\lambda_{r, v}$ by

$$
\begin{aligned}
& \text { if } r \neq v, \text { then } \lambda_{r, v}= \begin{cases}q^{-1} & \text { if } i=j \text { or } \alpha=\beta, \\
1 & \text { otherwise, }\end{cases} \\
& \text { if } r=v, \text { then } \lambda_{r, v}=q^{-2} .
\end{aligned}
$$

Recall that $R$ can be written as an iterated Ore extension

$$
R=\mathbb{C}\left[Y_{1,1}\right] \cdots\left[Y_{m, p} ; \sigma_{m, p}, \delta_{m, p}\right]
$$

where the indices are increasing for $\leqslant_{\mathrm{s}}$ and where, for $(1,2) \leqslant_{\mathrm{s}} r=(j, \beta) \leqslant_{\mathrm{s}}(m, p), \sigma_{r}$ is a $\mathbb{C}$-algebra automorphism and $\delta_{r}$ a $\mathbb{C}$-linear $\sigma_{r}$-derivation such that, for $(1,1) \leqslant_{\mathrm{s}} v=$ $(i, \alpha)<_{\mathrm{s}} r=(j, \beta)$,

$$
\begin{aligned}
\sigma_{r}\left(Y_{v}\right) & =\lambda_{r, v} Y_{v}, \\
\delta_{r}\left(Y_{v}\right) & = \begin{cases}-\left(q-q^{-1}\right) Y_{i, \beta} Y_{j, \alpha} & \text { if } i<j \text { and } \alpha<\beta, \\
0 & \text { otherwise. }\end{cases}
\end{aligned}
$$

In $[\mathbf{1 0}, \S 2.1]$, we have shown that the theory of deleting derivations (see $[\mathbf{2}]$ ) can be applied to the iterated Ore extension $R=\mathbb{C}\left[Y_{1,1}\right] \cdots\left[Y_{m, p} ; \sigma_{m, p}, \delta_{m, p}\right]$. The corresponding algorithm is called the standard deleting-derivations algorithm. It consists of the construction, for each $r \in E_{\mathrm{s}}$, of a family $\left(Y_{i, \alpha}^{(r)_{\mathrm{s}}}\right)_{(i, \alpha) \in[1, m \rrbracket \times \llbracket 1, p \rrbracket}$ of elements of $F=\operatorname{Fract}(R)$, defined as follows. 
(i) If $r=(m, p+1)$, then $Y_{i, \alpha}^{(m, p+1)_{\mathrm{s}}}=Y_{i, \alpha}$ for all $(i, \alpha) \in \llbracket 1, m \rrbracket \times \llbracket 1, p \rrbracket$.

(ii) Assume that $r=(j, \beta)<_{\mathrm{s}}(m, p+1)$ and that the $Y_{i, \alpha}^{\left(r^{+\mathrm{s}}\right)_{\mathrm{s}}}((i, \alpha) \in \llbracket 1, m \rrbracket \times \llbracket 1, p \rrbracket)$ are already known. For convenience of notation, we set

$$
Y_{i, \alpha}^{\left(r^{+}\right)_{\mathrm{s}}}=Y_{i, \alpha}^{\left(r^{+\mathrm{s}}\right)_{\mathrm{s}}} \quad \text { for }(i, \alpha) \in \llbracket 1, m \rrbracket \times \llbracket 1, p \rrbracket .
$$

If $(i, \alpha) \in \llbracket 1, m \rrbracket \times \llbracket 1, p \rrbracket$, then

$$
Y_{i, \alpha}^{(r)_{\mathrm{s}}}= \begin{cases}Y_{i, \alpha}^{\left(r^{+}\right)_{\mathrm{s}}}-Y_{i, \beta}^{\left(r^{+}\right)_{\mathrm{s}}}\left(Y_{j, \beta}^{\left(r^{+}\right)_{\mathrm{s}}}\right)^{-1} Y_{j, \alpha}^{\left(r^{+}\right)_{\mathrm{s}}} & \text { if } i<j \text { and } \alpha<\beta \\ Y_{i, \alpha}^{\left(r^{+}\right)_{\mathrm{s}}} & \text { otherwise. }\end{cases}
$$

Notation 2.3. Let $r \in E_{\mathrm{s}}$. We denote by $R^{(r)_{\mathrm{s}}}$ the subalgebra of $F=\operatorname{Fract}(R)$ generated by the $Y_{i, \alpha}^{(r)_{\mathrm{s}}}((i, \alpha) \in \llbracket 1, m \rrbracket \times \llbracket 1, p \rrbracket)$, that is,

$$
R^{(r)_{\mathrm{s}}}=\mathbb{C}\left\langle Y_{i, \alpha}^{(r)_{\mathrm{s}}} \mid(i, \alpha) \in \llbracket 1, m \rrbracket \times \llbracket 1, p \rrbracket\right\rangle .
$$

Remark 2.4. Let $r \in E_{\mathrm{s}}$ with $r \neq(m, p+1)$. We will often drop a subscript and write $R^{\left(r^{+}\right)_{\mathrm{s}}}$ for $R^{\left(r^{+\mathrm{s}}\right)_{\mathrm{s}}}$.

Notation 2.5. We set $\bar{R}_{\mathrm{s}}=R^{(1,2)_{\mathrm{s}}}$ and $T_{i, \alpha}=Y_{i, \alpha}^{(1,2)_{\mathrm{s}}}$ for all $(i, \alpha) \in \llbracket 1, m \rrbracket \times \llbracket 1, p \rrbracket$.

Let $(j, \beta) \in E_{\mathrm{s}}$ with $(j, \beta) \neq(m, p+1)$. The theory of deleting derivations allows us to construct embeddings $\varphi_{(j, \beta)_{\mathrm{s}}}: \operatorname{Spec}\left(R^{(j, \beta)_{\mathrm{s}}^{+}}\right) \rightarrow \operatorname{Spec}\left(R^{(j, \beta)_{\mathrm{s}}}\right)$ (see $\left.[2, \S 4.3]\right)$. By composition, we obtain an embedding $\varphi_{\mathrm{s}}: \operatorname{Spec}(R) \rightarrow \operatorname{Spec}\left(\bar{R}_{\mathrm{s}}\right)$, which is called the canonical embedding. Now to describe the $\operatorname{set} \mathcal{H}$ - $\operatorname{Spec}(R)$ we just have to determine its canonical image $\varphi_{\mathrm{s}}(\mathcal{H}-\operatorname{Spec}(R))$. To do this, as in [3, Conventions 3.2.1], we introduce some conventions and notation.

\section{Conventions 2.6.}

(i) Let $v=(l, \gamma) \in \llbracket 1, m \rrbracket \times \llbracket 1, p \rrbracket$.

(a) The set $C_{v}=\{(i, \gamma) \mid 1 \leqslant i \leqslant l\} \subset \llbracket 1, m \rrbracket \times \llbracket 1, p \rrbracket$ is called the truncated column with extremity $v$.

(b) The set $L_{v}=\{(l, \alpha) \mid 1 \leqslant \alpha \leqslant \gamma\} \subset \llbracket 1, m \rrbracket \times \llbracket 1, p \rrbracket$ is called the truncated row with extremity $v$.

(ii) $W$ denotes the set of all the subsets in $\llbracket 1, m \rrbracket \times \llbracket 1, p \rrbracket$ which are a union of truncated rows and columns.

Notation 2.7. Given $w \in W, K_{w}$ denotes the ideal in $\bar{R}_{\mathrm{s}}$ generated by the $T_{i, \alpha}$ such that $(i, \alpha) \in w$. (Recall that $K_{w}$ is a completely prime ideal in the quantum affine space $\bar{R}_{\mathrm{s}}($ see $[\mathbf{9}, \S 2.1])$.)

The following result is proved in the same manner as [3, Corollaire 3.2.1]. 


\section{Proposition 2.8.}

(i) Given $w \in W$, there exists a (unique) $\mathcal{H}$-invariant (completely) prime ideal $J_{w}$ in $R$ such that $\varphi_{\mathrm{s}}\left(J_{w}\right)=K_{w}$.

(ii) $\mathcal{H}-\operatorname{Spec}(R)=\left\{J_{w} \mid w \in W\right\}$.

\section{The factor ring $R / J_{w}$}

In this section, $K$ denotes a $\mathbb{C}$-algebra which is also a skew field. Except where stated otherwise, all matrices considered have their entries in $K$.

\section{Definition 3.1 (see Chapter 4 in [12]).}

(i) Let $u$ and $v$ be two positive integers and let $M=\left(x_{i, \alpha}\right)_{(i, \alpha) \in \llbracket 1, u \rrbracket \times \llbracket 1, v \rrbracket}$ be an $u \times v$ matrix. We say that $M$ is a q-quantum matrix if the following relations hold between the entries of $M$ :

$$
\begin{aligned}
& x_{i, \beta} x_{i, \alpha}=q^{-1} x_{i, \alpha} x_{i, \beta} \quad(1 \leqslant i \leqslant u, 1 \leqslant \alpha<\beta \leqslant v), \\
& x_{j, \alpha} x_{i, \alpha}=q^{-1} x_{i, \alpha} x_{j, \alpha} \quad(1 \leqslant i<j \leqslant u, 1 \leqslant \alpha \leqslant v), \\
& x_{j, \beta} x_{i, \alpha}=x_{i, \alpha} x_{j, \beta} \quad(1 \leqslant i<j \leqslant u, 1 \leqslant \beta<\alpha \leqslant v), \\
& x_{j, \beta} x_{i, \alpha}=x_{i, \alpha} x_{j, \beta}-\left(q-q^{-1}\right) x_{i, \beta} x_{j, \alpha} \quad(1 \leqslant i<j \leqslant u, 1 \leqslant \alpha<\beta \leqslant v) .
\end{aligned}
$$

(ii) Let $n$ be a positive integer and let $M=\left(x_{i, \alpha}\right)_{(i, \alpha) \in \llbracket 1, n \rrbracket^{2}}$ be a square $q$-quantum matrix. The quantum determinant of $M$ is defined by

$$
\operatorname{det}_{q}(M)=\sum_{\sigma \in S_{n}}(-q)^{l(\sigma)} x_{1, \sigma(1)} \cdots x_{n, \sigma(n)},
$$

where $l(\sigma)$ denotes the length of the $n$-permutation $\sigma$.

(iii) Let $M=\left(x_{i, \alpha}\right)_{(i, \alpha) \in \llbracket 1, m \rrbracket \times \llbracket 1, p \rrbracket}$ be a $q$-quantum matrix. The quantum determinant of a square sub-matrix of $M$ is called a quantum minor of $M$.

Definition 3.2. Let $M=\left(x_{i, \alpha}\right)_{(i, \alpha) \in \llbracket 1, m \rrbracket \times \llbracket 1, p \rrbracket}$ be an $m \times p$ matrix and let $(j, \beta) \in E_{\mathrm{s}}$. We say that $M$ is a $(j, \beta)_{\mathrm{s}}$-q-quantum matrix if the following relations hold between the entries of $M$.

If

$$
\left(\begin{array}{ll}
x & y \\
z & t
\end{array}\right)
$$

is any $2 \times 2$ sub-matrix of $M$, then

(i) $y x=q^{-1} x y, z x=q^{-1} x z, z y=y z, t y=q^{-1} y t, t z=q^{-1} z t$;

(ii) if $t=x_{v}$, then $\left\{\begin{array}{lll}v \geqslant_{\mathrm{s}}(j, \beta) & \Longrightarrow t x=x t, \\ v<_{\mathrm{s}}(j, \beta) & \Longrightarrow t x=x t-\left(q-q^{-1}\right) y z \text {. }\end{array}\right.$ 
Conventions 3.3 (see Convention 4.1.1 in [3] and Conventions 2.2.3 in [10]). Let

$$
M=\left(x_{i, \alpha}\right)_{(i, \alpha) \in \llbracket 1, m \rrbracket \times \llbracket 1, p \rrbracket}
$$

be a $q$-quantum matrix. As $r$ runs over the set $E_{\mathrm{s}}$, we define matrices

$$
M^{(r)_{\mathrm{s}}}=\left(x_{i, \alpha}^{(r)_{\mathrm{s}}}\right)_{(i, \alpha) \in \llbracket 1, m \rrbracket \times \llbracket 1, p \rrbracket}
$$

as follows.

(i) If $r=(m, p+1)$, then the entries of the matrix $M^{(m, p+1)_{\mathrm{s}}}$ are defined by $x_{i, \alpha}^{(m, p+1)_{\mathrm{s}}}=$ $x_{i, \alpha}$ for all $(i, \alpha) \in \llbracket 1, m \rrbracket \times \llbracket 1, p \rrbracket$.

(ii) Assume that $r=(j, \beta) \in E_{\mathrm{s}} \backslash\{(m, p+1)\}$ and that the matrix $M^{\left(r^{+\mathrm{s}}\right)_{\mathrm{s}}}$ is already known. For convenience of notation, we set $M^{\left(r^{+}\right)_{\mathrm{s}}}=M^{\left(r^{+\mathrm{s}}\right)_{\mathrm{s}}}$ and $x_{i, \alpha}^{\left(r^{+}\right)_{\mathrm{s}}}=x_{i, \alpha}^{\left(r^{+\mathrm{s}}\right)_{\mathrm{s}}}$ for each $(i, \alpha) \in \llbracket 1, m \rrbracket \times \llbracket 1, p \rrbracket$. The entries $x_{i, \alpha}^{(r)_{\mathrm{s}}}$ of the matrix $M^{(r)_{\mathrm{s}}}$ are defined as follows.

(a) If $x_{j, \beta}^{\left(r^{+}\right)_{\mathrm{s}}}=0$, then $x_{i, \alpha}^{(r)_{\mathrm{s}}}=x_{i, \alpha}^{\left(r^{+}\right)_{\mathrm{s}}}$ for all $(i, \alpha) \in \llbracket 1, m \rrbracket \times \llbracket 1, p \rrbracket$.

(b) If $x_{j, \beta}^{\left(r^{+}\right)_{s}} \neq 0$ and $(i, \alpha) \in \llbracket 1, m \rrbracket \times \llbracket 1, p \rrbracket$, then

$$
x_{i, \alpha}^{(r)_{\mathrm{s}}}= \begin{cases}x_{i, \alpha}^{\left(r^{+}\right)_{\mathrm{s}}}-x_{i, \beta}^{\left(r^{+}\right)_{\mathrm{s}}}\left(x_{j, \beta}^{\left(r^{+}\right)_{\mathrm{s}}}\right)^{-1} x_{j, \alpha}^{\left(r^{+}\right)_{\mathrm{s}}} & \text { if } i<j \text { and } \alpha<\beta, \\ x_{i, \alpha}^{\left(r^{+}\right)_{\mathrm{s}}} & \text { otherwise. }\end{cases}
$$

We say that $M^{(r)_{s}}$ is the matrix obtained from $M$ by applying the standard deletingderivations algorithm at step $r$.

(iii) If $r=(1,2)$, we set $t_{i, \alpha}=x_{i, \alpha}^{(1,2)_{\mathrm{s}}}$ for all $(i, \alpha) \in \llbracket 1, m \rrbracket \times \llbracket 1, p \rrbracket$.

Note that our definitions of $q$-quantum matrix and $(j, \beta)_{\mathrm{s}}-q$-quantum matrix slightly differ from those of [1] (see [1, Definitions III.1.1 and III.1.3]). Because of this, we must interchange $q$ and $q^{-1}$ whenever carrying over a result of $[\mathbf{1}]$.

Lemma 3.4. Let $(j, \beta) \in E_{\mathrm{s}}$. If $M=\left(x_{i, \alpha}\right)_{(i, \alpha) \in \llbracket 1, m \rrbracket \times \llbracket 1, p \rrbracket}$ is a q-quantum matrix, then the matrix $M^{(j, \beta)_{\mathrm{s}}}$ is $(j, \beta)_{\mathrm{s}}-q$-quantum.

Proof. This lemma is proved in the same manner as [1, Proposition III.2.3.1].

The formulae of Conventions 3.3 allow us to express the entries of $M^{\left(r^{+}\right)_{\mathrm{s}}}$ in terms of those of $M^{(r)_{s}}$.

Proposition 3.5 (restoration algorithm). Let $M=\left(x_{i, \alpha}\right)_{(i, \alpha) \in \llbracket 1, m \rrbracket \times \llbracket 1, p \rrbracket}$ be a $q$ quantum matrix and let $r=(j, \beta) \in E_{\mathrm{s}}$ with $r \neq(m, p+1)$. 
(1) If $x_{j, \beta}^{(r)_{\mathrm{s}}}=0$, then $x_{i, \alpha}^{\left(r^{+}\right)_{\mathrm{s}}}=x_{i, \alpha}^{(r)_{\mathrm{s}}}$ for all $(i, \alpha) \in \llbracket 1, m \rrbracket \times \llbracket 1, p \rrbracket$.

(2) If $x_{j, \beta}^{(r)_{\mathrm{s}}} \neq 0$ and $(i, \alpha) \in \llbracket 1, m \rrbracket \times \llbracket 1, p \rrbracket$, then

$$
x_{i, \alpha}^{\left(r^{+}\right)_{\mathrm{s}}}= \begin{cases}x_{i, \alpha}^{(r)_{\mathrm{s}}}+x_{i, \beta}^{(r)_{\mathrm{s}}}\left(x_{j, \beta}^{(r)_{\mathrm{s}}}\right)^{-1} x_{j, \alpha}^{(r)_{\mathrm{s}}} & \text { if } i<j \text { and } \alpha<\beta \\ x_{i, \alpha}^{(r)_{\mathrm{s}}} & \text { otherwise. }\end{cases}
$$

We now come back to the $\mathcal{H}$-invariant prime ideals $J_{w}$ of $R$ (see the notation of $\S 2$ ). The aim of the rest of this section is to study the effect of the standard deleting-derivations algorithm on the matrix whose entries are $y_{i, \alpha}=Y_{i, \alpha}+J_{w}((i, \alpha) \in \llbracket 1, m \rrbracket \times \llbracket 1, p \rrbracket)$.

Notation 3.6. Let $w \in W$.

(i) Set $R_{w}=R / J_{w}$. It follows from $\left[\mathbf{2}\right.$, Lemme 5.3.3] that $R_{w}$ and $\bar{R}_{\mathrm{s}} / K_{w}$ are two Noetherian algebras with no zero-divisors and which have the same skew field of fractions. We set $F_{w}=\operatorname{Fract}\left(R_{w}\right)=\operatorname{Fract}\left(\bar{R}_{\mathrm{s}} / K_{w}\right)$.

(ii) If $(i, \alpha) \in \llbracket 1, m \rrbracket \times \llbracket 1, p \rrbracket$, then $y_{i, \alpha}$ denotes the element of $R_{w}$ defined by $y_{i, \alpha}=$ $Y_{i, \alpha}+J_{w}$.

(iii) We denote by $M_{w}$ the matrix, with entries in the $\mathbb{C}$-algebra $F_{w}$, defined by

$$
M_{w}=\left(y_{i, \alpha}\right)_{(i, \alpha) \in \llbracket 1, m \rrbracket \times \llbracket 1, p \rrbracket} .
$$

Let $w \in W$. Since $\mathcal{Y}=\left(Y_{i, \alpha}\right)_{(i, \alpha) \in \llbracket 1, m \rrbracket \times \llbracket 1, p \rrbracket}$ is a $q$-quantum matrix, $M_{w}$ is also a $q$ quantum matrix. Thus, we can apply the standard deleting-derivations algorithm to $M_{w}$ (see Conventions 3.3 with $K=F_{w}$ ) and if we still denote $t_{i, \alpha}=y_{i, \alpha}^{(1,2)_{s}}$ for $(i, \alpha) \in$ $\llbracket 1, m \rrbracket \times \llbracket 1, p \rrbracket$, we get the following theorem.

\section{Theorem 3.7.}

(i) $M_{w}^{(1,2)_{\mathrm{s}}}$ is $(1,2)_{\mathrm{s}}-q$-quantum.

(ii) $t_{i, \alpha}=0$ if and only if $(i, \alpha) \in w$.

(iii) There is a $\mathbb{C}$-algebra isomorphism from $\mathbb{C}\left\langle t_{i, \alpha} \mid(i, \alpha) \notin w\right\rangle$ onto the subalgebra $\mathbb{C}\left\langle T_{i, \alpha} \mid(i, \alpha) \notin w\right\rangle$ of $\bar{R}_{\mathrm{s}}$, which sends $t_{i, \alpha}$ onto $T_{i, \alpha}$ for each $(i, \alpha) \notin w$.

Proof. The first point follows from Lemma 3.4. By [2, Propositions 5.4.1 and 5.4.2], there exists a $\mathbb{C}$-algebra homomorphism $f_{(1,2)}: \bar{R}_{\mathrm{s}} \rightarrow F_{w}$ such that $f_{(1,2)}\left(T_{i, \alpha}\right)=t_{i, \alpha}$ for $(i, \alpha) \in \llbracket 1, m \rrbracket \times \llbracket 1, p \rrbracket$. Its kernel is $K_{w}$ and its image is the subalgebra of $F_{w}$ generated by the $t_{i, \alpha}$ with $(i, \alpha) \in \llbracket 1, m \rrbracket \times \llbracket 1, p \rrbracket$. Hence $t_{i, \alpha}=0$ if and only if $T_{i, \alpha} \in K_{w}$, that is, if and only if $(i, \alpha) \in w$, and

$$
\mathbb{C}\left\langle t_{i, \alpha} \mid(i, \alpha) \notin w\right\rangle \simeq \bar{R}_{\mathrm{s}} / K_{w} \simeq \mathbb{C}\left\langle T_{i, \alpha} \mid(i, \alpha) \notin w\right\rangle .
$$




\section{An algorithm which computes a generating set for $J_{w}$}

Input. Fix $w \in W$. Denote by $M_{w}=\left(y_{i, \alpha}\right)_{(i, \alpha) \in \llbracket 1, m \rrbracket \times \llbracket 1, p \rrbracket}$ the matrix whose entries are

$$
y_{i, \alpha}=Y_{i, \alpha}+J_{w} \quad((i, \alpha) \in \llbracket 1, m \rrbracket \times \llbracket 1, p \rrbracket) .
$$

It follows from Theorem 3.7 that $M_{w}^{(1,2)_{\mathrm{s}}}=\left(t_{i, \alpha}\right)_{(i, \alpha) \in \llbracket 1, m \rrbracket \times \llbracket 1, p \rrbracket}$ is a $(1,2)_{\mathrm{s}}$ - $q$-quantum matrix whose entries have the following properties.

(i) $t_{i, \alpha}=0$ if and only if $(i, \alpha) \in w$.

(ii) There is an isomorphism from $\mathbb{C}\left\langle t_{i, \alpha} \mid(i, \alpha) \notin w\right\rangle$ onto $\mathbb{C}\left\langle T_{i, \alpha} \mid(i, \alpha) \notin w\right\rangle$, which sends $t_{i, \alpha}$ onto $T_{i, \alpha}((i, \alpha) \notin w)$.

Step 1: restoration of $M_{\boldsymbol{w}}$. Starting with the matrix $M_{w}^{(1,2)_{s}}$, we compute the matrix

$$
M_{w}=\left(y_{i, \alpha}\right)_{(i, \alpha) \in \llbracket 1, m \rrbracket \times \llbracket 1, p \rrbracket}
$$

by using the restoration algorithm (see Proposition 3.5). This is a q-quantum matrix with entries in the McConnell-Pettit algebra $\mathbb{C}\left\langle t_{i, \alpha}^{ \pm 1} \mid(i, \alpha) \notin w\right\rangle$.

\section{Step 2: we calculate all quantum minors of $M_{w}$.}

Result. Let

$$
X_{w}=\left\{(I, \Lambda)|I \subseteq \llbracket 1, m \rrbracket, \Lambda \subseteq \llbracket 1, p \rrbracket,| I|=| \Lambda \mid \text { and } \operatorname{det}_{q}\left(y_{i, \alpha}\right)_{(i, \alpha) \in I \times \Lambda}=0\right\} .
$$

Then $J_{w}$ is generated, as right and left ideal, by the quantum minors $\operatorname{det}_{q}\left(Y_{i, \alpha}\right)_{(i, \alpha) \in I \times \Lambda}$ with $(I, \Lambda) \in X_{w}$.

Proof. This is immediate from [10, Théorème 3.7.2].

Example 4.1. Assume that $m=p=3$. If this algorithm is applied to $w=\{(1,1)$, $(1,3),(2,1),(2,2)\}$, one can show that the two-sided ideal in $O_{q}\left(\mathcal{M}_{3}(\mathbb{C})\right)$ generated by

$$
\begin{aligned}
Y_{1,3}, \quad \operatorname{det}_{q}\left(\begin{array}{ll}
Y_{1,1} & Y_{1,2} \\
Y_{2,1} & Y_{2,2}
\end{array}\right), \quad \operatorname{det}_{q}\left(\begin{array}{ll}
Y_{1,1} & Y_{1,2} \\
Y_{3,1} & Y_{3,2}
\end{array}\right), \\
\operatorname{det}_{q}\left(\begin{array}{ll}
Y_{2,1} & Y_{2,2} \\
Y_{3,1} & Y_{3,2}
\end{array}\right), \quad \operatorname{det}_{q}\left(\begin{array}{ll}
Y_{2,1} & Y_{2,3} \\
Y_{3,1} & Y_{3,3}
\end{array}\right) \quad \text { and } \quad \operatorname{det}_{q}\left(\begin{array}{ll}
Y_{2,2} & Y_{2,3} \\
Y_{3,2} & Y_{3,3}
\end{array}\right)
\end{aligned}
$$

is (completely) prime. In the more general case where we just assume that $q \in \mathbb{C}^{*}$ is not a root of unity, this result was proved by Goodearl and Lenagan (see $[\mathbf{6}, \S 7.2]$ ) by using different methods. 


\section{The last-column deleting-derivations algorithm}

The aim of this section is to define a new deleting-derivations algorithm which will allow us to show that certain sets of quantum minors generate prime ideals of $R$. We shall only use it when $m$ and $p$ are greater than or equal to 3 , so for the remainder of this section, we assume that $\min (m, p) \geqslant 3$ (although most of the following results are still true when $m=2$ or $p=2$ ).

Definition 5.1. Define the relation $\leqslant_{\mathrm{dc}}$ by

$(i, \alpha) \leqslant \mathrm{dc}(j, \beta)$

$\Longleftrightarrow[(\alpha=\beta=p$ and $i \leqslant j)$ or $(\beta=p$ and $\alpha<p)$ or $(\alpha, \beta<p$ and $(i, \alpha) \leqslant \mathrm{s}(j, \beta))]$.

This defines a total ordering on $\llbracket 1, m \rrbracket \times \llbracket 1, p \rrbracket \cup\{(m+1, p)\}$ that we will call the lastcolumn ordering on $\llbracket 1, m \rrbracket \times \llbracket 1, p \rrbracket \cup\{(m+1, p)\}$.

\section{Notation 5.2.}

(i) We set $E_{\mathrm{dc}}=(\llbracket 1, m \rrbracket \times \llbracket 1, p \rrbracket \cup\{(m+1, p\}) \backslash\{(1,1)\}$.

(ii) Let $(j, \beta) \in E_{\mathrm{dc}}$. If $(j, \beta) \neq(m+1, p)$, denote by $(j, \beta)^{+\mathrm{dc}}$ the smallest element (relative to $\leqslant_{\mathrm{dc}}$ ) of the set $\left\{(i, \alpha) \in E_{\mathrm{dc}} \mid(j, \beta)<_{\mathrm{dc}}(i, \alpha)\right\}$.

Using [2, Propositions 6.1.1 and 6.1.2], we get the following theorem.

\section{Theorem 5.3.}

(1) $R$ can be written as an iterated Ore extension

$$
R=\mathbb{C}\left[Y_{1,1}\right] \cdots\left[Y_{m, p-1} ; \sigma_{m, p-1}^{\prime}, \delta_{m, p-1}^{\prime}\right]\left[Y_{1, p} ; \sigma_{1, p}^{\prime}, \delta_{1, p}^{\prime}\right] \cdots\left[Y_{m, p} ; \sigma_{m, p}^{\prime}, \delta_{m, p}^{\prime}\right],
$$

where the indices are increasing for $\leqslant_{\mathrm{dc}}$ and where, for $(1,2) \leqslant_{\mathrm{dc}} r=(j, \beta) \leqslant_{\mathrm{dc}}$ $(m, p), \sigma_{r}^{\prime}$ is a $\mathbb{C}$-algebra automorphism and $\delta_{r}^{\prime}$ a $\mathbb{C}$-linear $\sigma_{r}^{\prime}$-derivation such that, for $(1,1) \leqslant \mathrm{dc} v=(i, \alpha)<_{\mathrm{dc}} r=(j, \beta)$,

$$
\begin{aligned}
& \sigma_{r}^{\prime}\left(Y_{v}\right)=\lambda_{r, v} Y_{v} \quad\left(\lambda_{r, v}\right. \text { was defined in Notation 2.2); } \\
& \delta_{r}^{\prime}\left(Y_{v}\right)= \begin{cases}-\left(q-q^{-1}\right) Y_{i, \beta} Y_{j, \alpha} & \text { if } i<j \text { and } \alpha<\beta, \\
0 & \text { otherwise. }\end{cases}
\end{aligned}
$$

(2) $R$ satisfies Conventions 3.1 of [2] with $q_{r}=q^{-2}$ for any $r \in \llbracket 1, m \rrbracket \times \llbracket 1, p \rrbracket$.

(3) If $r \in \llbracket 1, m \rrbracket \times \llbracket 1, p \rrbracket \backslash\{(1,1)\}$, there exists $h_{r}^{\prime} \in \mathcal{H}$ such that $h_{r}^{\prime} . Y_{v}=\lambda_{r, v} Y_{v}$ for $v \in \llbracket 1, m \rrbracket \times \llbracket 1, p \rrbracket$. Thus, $R$ satisfies Hypotheses 4.1 .2 of $[\mathbf{2}]$ with the group $\mathcal{H}$.

It follows from the previous theorem that the theory of deleting derivations (see [2]) can be applied to the iterated Ore extension $R=\mathbb{C}\left[Y_{1,1}\right] \cdots\left[Y_{m, p} ; \sigma_{m, p}^{\prime}, \delta_{m, p}^{\prime}\right]$. The corresponding algorithm is called the last-column deleting-derivations algorithm. 
Let $r=(j, \beta) \in \llbracket 1, m \rrbracket \times \llbracket 1, p \rrbracket$ with $(1,1)<_{\mathrm{dc}} r$. Denote by $B$ the subalgebra of $R$ generated by the $Y_{v}$ with $v \in \llbracket 1, m \rrbracket \times \llbracket 1, p \rrbracket$ and $(1,1) \leqslant \mathrm{dc} v<_{\mathrm{dc}} r$, and let $C$ be the subalgebra of $R$ generated by $B$ and $Y_{r}$. It follows from Theorem 5.3 that $C$ is the (left) Ore extension $B\left[Y_{r} ; \sigma_{r}^{\prime}, \delta_{r}^{\prime}\right]$, and that, in $F=\operatorname{Fract}(R)$, we have

$$
\sum_{k=0}^{+\infty} \frac{\left(1-q^{-2}\right)^{-k}}{[k] !_{q^{-2}}} \lambda_{r, v}^{-k} \delta_{r}^{\prime k}\left(Y_{v}\right) Y_{r}^{-k}= \begin{cases}Y_{v}-Y_{i, \beta} Y_{j, \beta}^{-1} Y_{j, \alpha} & \text { if } i<j \text { and } \alpha<\beta, \\ Y_{v} & \text { otherwise }\end{cases}
$$

where $[k] !_{q^{-2}}=[0]_{q^{-2}} \times \cdots \times[k]_{q^{-2}}$ with $[0]_{q^{-2}}=1$ and $[i]_{q^{-2}}=1+q^{-2}+\cdots+q^{-2(i-1)}$ if $i$ is a positive integer.

Hence, the last-column deleting-derivations algorithm consists of the construction, for each $r \in E_{\mathrm{dc}}$, of a family $\left(Y_{i, \alpha}^{(r)_{\mathrm{dc}}}\right)_{(i, \alpha) \in \llbracket 1, m \rrbracket \times \llbracket 1, p \rrbracket}$ of elements of $F=\operatorname{Fract}(R)$, defined as follows.

(1) If $r=(m+1, p)$, then $Y_{i, \alpha}^{(m+1, p)_{\mathrm{dc}}}=Y_{i, \alpha}$ for all $(i, \alpha) \in \llbracket 1, m \rrbracket \times \llbracket 1, p \rrbracket$.

(2) Assume that $r=(j, \beta)<_{\mathrm{dc}}(m+1, p)$ and that the

$$
\left.Y_{i, \alpha}^{\left(r^{+} \mathrm{dc}\right)}\right)_{\mathrm{dc}} \quad((i, \alpha) \in \llbracket 1, m \rrbracket \times \llbracket 1, p \rrbracket)
$$

are already known. For convenience of notation, we set $\left.Y_{i, \alpha}^{\left(r^{+}\right)}\right)_{\mathrm{dc}}=Y_{i, \alpha}^{\left(r^{+} \mathrm{dc}\right) \mathrm{dc}}$ for $(i, \alpha) \in \llbracket 1, m \rrbracket \times \llbracket 1, p \rrbracket$.

If $(i, \alpha) \in \llbracket 1, m \rrbracket \times \llbracket 1, p \rrbracket$, then

$$
Y_{i, \alpha}^{(r) \mathrm{dc}}= \begin{cases}Y_{i, \alpha}^{\left(r^{+}\right)_{\mathrm{dc}}}-Y_{i, \beta}^{\left(r^{+}\right) \mathrm{dc}}\left(Y_{j, \beta}^{\left(r^{+}\right) \mathrm{dc}}\right)^{-1} Y_{j, \alpha}^{\left(r^{+}\right) \mathrm{dc}} & \text { if } i<j \text { and } \alpha<\beta, \\ Y_{i, \alpha}^{\left(r^{+}\right){ }_{\mathrm{dc}}} & \text { otherwise. }\end{cases}
$$

\section{A link between the standard and last-column deleting-derivations algorithms}

Throughout this section, we use the following conventions.

(i) We assume that $\min (m, p) \geqslant 3$.

(ii) $K$ denotes a $\mathbb{C}$-algebra which is also a skew field. All the matrices considered have their entries in $K$.

Conventions 6.1. Let $M=\left(x_{i, \alpha}\right)_{(i, \alpha) \in[1, m] \times \llbracket 1, p \rrbracket}$ be a $q$-quantum matrix. As $r$ runs over the set $E_{\mathrm{dc}}$, we define matrices

$$
M^{(r)_{\mathrm{dc}}}=\left(x_{i, \alpha}^{(r)_{\mathrm{dc}}}\right)_{(i, \alpha) \in \llbracket 1, m \rrbracket \times \llbracket 1, p \rrbracket}
$$

as follows.

(1) If $r=(m+1, p)$, then the entries of the matrix $M^{(m+1, p)_{\mathrm{dc}}}$ are defined by $x_{i, \alpha}^{(m+1, p))_{\mathrm{dc}}}=x_{i, \alpha}$ for all $(i, \alpha) \in \llbracket 1, m \rrbracket \times \llbracket 1, p \rrbracket$. 
(2) Assume that $r=(j, \beta) \in E_{\mathrm{dc}} \backslash\{(m+1, p)\}$ and that the matrix $M^{\left(r^{+} \mathrm{dc}\right)}$ dc is already known. We set

$$
M^{\left(r^{+}\right)_{\mathrm{dc}}}=M^{\left(r^{+} \mathrm{dc}\right)_{\mathrm{dc}}} \quad \text { and } \quad x_{i, \alpha}^{\left(r^{+}\right)_{\mathrm{dc}}}=x_{i, \alpha}^{\left(r^{+} \mathrm{dc}\right)} \quad \text { for each }(i, \alpha) \in \llbracket 1, m \rrbracket \times \llbracket 1, p \rrbracket .
$$

The entries $x_{i, \alpha}^{(r)_{\mathrm{dc}}}$ of the matrix $M^{(r)_{\mathrm{dc}}}$ are defined as follows.

(a) If $x_{j, \beta}^{\left(r^{+}\right)_{\mathrm{dc}}}=0$, then $x_{i, \alpha}^{(r)_{\mathrm{dc}}}=x_{i, \alpha}^{\left(r^{+}\right)_{\mathrm{dc}}}$ for all $(i, \alpha) \in \llbracket 1, m \rrbracket \times \llbracket 1, p \rrbracket$.

(b) If $x_{j, \beta}^{\left(r^{+}\right)_{\mathrm{dc}}} \neq 0$ and $(i, \alpha) \in \llbracket 1, m \rrbracket \times \llbracket 1, p \rrbracket$, then

$$
x_{i, \alpha}^{(r)_{\mathrm{dc}}}= \begin{cases}x_{i, \alpha}^{\left(r^{+}\right)_{\mathrm{dc}}}-x_{i, \beta}^{\left(r^{+}\right)_{\mathrm{dc}}}\left(x_{j, \beta}^{\left(r^{+}\right)_{\mathrm{dc}}}\right)^{-1} x_{j, \alpha}^{\left.\left(r^{+}\right)\right)_{\mathrm{dc}}} & \text { if } i<j \text { and } \alpha<\beta, \\ x_{i, \alpha}^{\left(r^{+}\right)_{\mathrm{dc}}} & \text { otherwise. }\end{cases}
$$

We say that $M^{(r)_{\mathrm{dc}}}$ is the matrix obtained from $M$ by applying the last-column deleting-derivations algorithm at step $r$.

Let $M=\left(x_{i, \alpha}\right)_{(i, \alpha) \in \llbracket 1, m \rrbracket \times \llbracket 1, p \rrbracket}$ be a $q$-quantum matrix. The following lemma is obvious.

\section{Lemma 6.2.}

(1) $M^{(m, p)_{\mathrm{s}}}=M^{(m, p)_{\mathrm{dc}}}$.

(2) If $(j, \beta) \in E_{\mathrm{s}} \cap E_{\mathrm{dc}}=\llbracket 1, m \rrbracket \times \llbracket 1, p \rrbracket \backslash\{(1,1)\}$, then

$$
\begin{array}{ll}
x_{m, \alpha}^{(j, \beta)_{\mathrm{s}}}=x_{m, \alpha}^{(j, \beta)_{\mathrm{dc}}}=x_{m, \alpha} & \text { for any } \alpha \in \llbracket 1, p \rrbracket, \\
x_{i, p}^{(j, \beta)_{\mathrm{s}}}=x_{i, p}^{(j, \beta)_{\mathrm{dc}}}=x_{i, p} & \text { for any } i \in \llbracket 1, m \rrbracket .
\end{array}
$$

Proposition 6.3. If $M=\left(x_{i, \alpha}\right)_{(i, \alpha) \in \llbracket 1, m \rrbracket \times \llbracket 1, p \rrbracket}$ is a q-quantum matrix, then $M^{(1,2)_{\mathrm{s}}}=$ $M^{(1,2) \mathrm{dc}}$.

Since the proof of this result is very technical, we just give a sketch.

Proof. First, if $i=m$ or $\alpha=p$, it follows from Lemma 6.2 that $x_{i, \alpha}^{(1,2)_{\mathrm{dc}}}=t_{i, \alpha}$. Now we assume that $i \leqslant m-1$ and $\alpha \leqslant p-1$. A decreasing induction shows that, if $j \in \llbracket 1, m+1 \rrbracket$, then

$$
x_{i, \alpha}=x_{i, \alpha}^{(j, p)_{\mathrm{dc}}}+\sum_{\substack{k=\max _{x_{k, p}(i+1, j)} \neq 0 \\ m}}^{m i, p} x_{k, p}^{-1} x_{k, \alpha}^{(j, p)_{\mathrm{dc}}} .
$$

In particular, for $j=1$, we obtain

$$
x_{i, \alpha}=x_{i, \alpha}^{(1, p)_{\mathrm{dc}}}+\sum_{\substack{k=i+1 \\ x_{k, p} \neq 0}}^{m} x_{i, p} x_{k, p}^{-1} x_{k, \alpha}^{(1, p)_{\mathrm{dc}}} .
$$


Now, we easily deduce the following equalities from (6.1):

$$
x_{i, \alpha}^{(m, p)_{\mathrm{s}}}=x_{i, \alpha}^{(1, p)_{\mathrm{dc}}}+\sum_{\substack{k=i+1 \\ x_{k, p} \neq 0}}^{m-1} x_{i, p} x_{k, p}^{-1} x_{k, \alpha}^{(1, p)_{\mathrm{dc}}}
$$

and

$$
x_{i, \alpha}^{(m, p-1)_{\mathrm{s}}}=x_{i, \alpha}^{(m, p-1)_{\mathrm{dc}}}+\sum_{\substack{k=i+1 \\ x_{k, p} \neq 0}}^{m-1} x_{i, p} x_{k, p}^{-1} x_{k, \alpha}^{(m, p-1)_{\mathrm{dc}}} .
$$

Next, by a decreasing induction (with respect to $\leqslant_{\mathrm{dc}}$ ), we can show that, if $(j, \beta) \in E_{\mathrm{dc}}$ with $(j, \beta) \leqslant \mathrm{dc}(m, p-1)$, then

$$
x_{i, \alpha}^{(j, \beta)_{\mathrm{s}}}=x_{i, \alpha}^{(j, \beta)_{\mathrm{dc}}}+\sum_{\substack{k=i+1 \\ x_{k, p} \neq 0}}^{j-1} x_{i, p} x_{k, p}^{-1} x_{k, \alpha}^{(j, \beta)_{\mathrm{dc}}} .
$$

For $(j, \beta)=(1,2)$, equality $(6.2)$ becomes $t_{i, \alpha}=x_{i, \alpha}^{(1,2)_{\mathrm{dc}}}$, and Proposition 6.3 follows.

Corollary 6.4. Let $M=\left(x_{i, \alpha}\right)_{(i, \alpha) \in \llbracket 1, m \rrbracket \times \llbracket 1, p \rrbracket}$ be a q-quantum matrix. Then

$$
x_{j, \beta}^{(j, \beta)_{\mathrm{dc}}^{+}}=t_{j, \beta} \quad \text { for any }(j, \beta) \in \llbracket 1, m \rrbracket \times \llbracket 1, p \rrbracket .
$$

Proof. Since

$$
x_{j, \beta}^{(j, \beta)_{\mathrm{dc}}^{+}}=x_{j, \beta}^{(1,2)_{\mathrm{dc}}} \quad \text { for any }(j, \beta) \in \llbracket 1, m \rrbracket \times \llbracket 1, p \rrbracket,
$$

this corollary is an immediate consequence of Proposition 6.3.

\section{The effect of the last-column deleting-derivations algorithm on quantum minors}

Throughout this section, we keep the conventions and notation of $\S 6$.

Definition 7.1. Let $M=\left(x_{i, \alpha}\right)_{(i, \alpha) \in[1, m \rrbracket \times[1, p]}$ be a matrix and let $(j, \beta) \in E_{\mathrm{dc}}$. We say that $M$ is a $(j, \beta)_{\mathrm{dc}}-q$-quantum matrix if the following relations hold between the entries of $M$.

If

$$
\left(\begin{array}{ll}
x & y \\
z & t
\end{array}\right)
$$

is any $2 \times 2$ sub-matrix of $M$, then

(1) $y x=q^{-1} x y, z x=q^{-1} x z, z y=y z, t y=q^{-1} y t, t z=q^{-1} z t$;

(2) if $t=x_{v}$, then $\begin{cases}v \geqslant_{\mathrm{dc}}(j, \beta) & \Longrightarrow t x=x t, \\ v<_{\mathrm{dc}}(j, \beta) & \Longrightarrow t x=x t-\left(q-q^{-1}\right) y z \text {. }\end{cases}$ 
Lemma 7.2. Let $(j, \beta) \in E_{\mathrm{dc}}$. If $M=\left(x_{i, \alpha}\right)_{(i, \alpha) \in \llbracket 1, m \rrbracket \times \llbracket 1, p \rrbracket}$ is a q-quantum matrix, then $M^{(j, \beta)_{\mathrm{dc}}}$ is a $(j, \beta)_{\mathrm{dc}}-q$-quantum matrix.

Proof. First, it follows from [2, Théorème 3.2.1] that the matrix

$$
\left(Y_{i, \alpha}^{(j, \beta)_{\mathrm{dc}}}\right)_{(i, \alpha) \in \llbracket 1, m \rrbracket \times \llbracket 1, p \rrbracket}
$$

(see $\S 5)$ is a $(j, \beta)_{\mathrm{dc}}-q$-quantum matrix. The rest of the proof is similar to $[\mathbf{1 0}$, Lemme 2.5.3].

The following result can be deduced easily from this lemma.

Corollary 7.3. Let $M$ be an $m \times p$-quantum matrix and let $(j, \beta) \in E_{\mathrm{dc}}$.

(1) If $\beta=p$, then

(a) the matrix obtained from $M^{(j, \beta)_{\mathrm{dc}}}$ by deleting the last column is q-quantum;

(b) the matrix obtained from $M^{(j, \beta)_{\mathrm{dc}}}$ by deleting the rows $j, \ldots, m$ is q-quantum $(j>1)$.

(2) If $\beta<p$, then

(a) the matrix obtained from $M^{(j, \beta)_{\mathrm{dc}}}$ by deleting the rows $j, \ldots, m$ and the last column is q-quantum $(j>1)$;

(b) the matrix obtained from $M^{(j, \beta)_{\mathrm{dc}}}$ by deleting the rows $j+1, \ldots, m$ and the columns $\beta, \ldots, p$ is q-quantum $(j, \beta>1)$.

Let $M=\left(x_{i, \alpha}\right)_{(i, \alpha) \in \llbracket 1, m \rrbracket \times \llbracket 1, p \rrbracket}$ be a $q$-quantum matrix. We now express the quantum minors of $M^{(j, \beta)_{\mathrm{dc}}^{+}}$in terms of those of $M^{(j, \beta)_{\mathrm{dc}}}$.

Notation 7.4. Let $M=\left(x_{i, \alpha}\right)_{(i, \alpha) \in \llbracket 1, m \rrbracket \times \llbracket 1, p \rrbracket}$ be a $q$-quantum matrix and let

$$
\delta=\operatorname{det}_{q}\left(x_{i, \alpha}\right) \underset{\substack{i=i_{1}, \ldots ., i_{l} \\ \alpha=\alpha_{1}, \ldots, \alpha_{l}}}{ }
$$

be an $l \times l$ quantum minor of $M\left(1 \leqslant l \leqslant \min (m, p), 1 \leqslant i_{1}<\cdots<i_{l} \leqslant m, 1 \leqslant \alpha_{1}<\right.$ $\left.\cdots<\alpha_{l} \leqslant p\right)$.

(1) (a) If $I$ is a non-empty subset of $\left\{i_{1}, \ldots, i_{l}\right\}$, we set $\hat{I}=\left\{i_{1}, \ldots, i_{l}\right\} \backslash I$. In the particular case where $I=\left\{i_{k}\right\}(k \in \llbracket 1, l \rrbracket)$, we set $\hat{i}_{k}=\hat{I}$.

(b) If $\Lambda$ is a non-empty subset of $\left\{\alpha_{1}, \ldots, \alpha_{l}\right\}$, we set $\bar{\Lambda}=\left\{\alpha_{1}, \ldots, \alpha_{l}\right\} \backslash \Lambda$. In the particular case where $\Lambda=\left\{\alpha_{k}\right\}(k \in \llbracket 1, l \rrbracket)$, we set $\bar{\alpha}_{k}=\bar{\Lambda}$.

(Observe that the set $\hat{I}$ (respectively, $\bar{\Lambda}$ ) depends on the set $\left\{i_{1}, \ldots, i_{l}\right\}$ (respectively, $\left.\left\{\alpha_{1}, \ldots, \alpha_{l}\right\}\right)$.) 
(2) If $(j, \beta) \in E_{\mathrm{dc}}$ is greater (relative to $\leqslant_{\mathrm{dc}}$ ) than the elements of $\left\{i_{1}, \ldots, i_{l}\right\} \times$ $\left\{\alpha_{1}, \ldots, \alpha_{l}\right\}$, it follows from Lemma 7.2 that the matrix

$$
\left(x_{i, \alpha}^{(j, \beta)_{\mathrm{dc}}}\right)_{(i, \alpha) \in\left\{i_{1}, \ldots, i_{l}\right\} \times\left\{\alpha_{1}, \ldots, \alpha_{l}\right\}}
$$

is $q$-quantum. We set

$$
\delta^{(j, \beta)_{\mathrm{dc}}}=\operatorname{det}_{q}\left(x_{i, \alpha}^{(j, \beta)_{\mathrm{dc}}}\right)_{(i, \alpha) \in\left\{i_{1}, \ldots, i_{l}\right\} \times\left\{\alpha_{1}, \ldots, \alpha_{l}\right\}} .
$$

(3) Let $I$ be a non-empty subset of $\left\{i_{1}, \ldots, i_{l}\right\}$ and let $\Lambda$ be a non-empty subset of $\left\{\alpha_{1}, \ldots, \alpha_{l}\right\}$ with $|I|=|\Lambda|$.

(a) We set $\delta_{\hat{I}, \bar{\Lambda}}=\operatorname{det}_{q}\left(x_{i, \alpha}\right)_{(i, \alpha) \in \hat{I} \times \bar{\Lambda}}$.

(b) If $(j, \beta) \in E_{\mathrm{dc}}$ is greater (relative to $\leqslant \mathrm{dc}$ ) than the elements of $\hat{I} \times \bar{\Lambda}$, it follows from Lemma 7.2 that the matrix $\left(x_{i, \alpha}^{(j, \beta)_{\mathrm{dc}}}\right)_{(i, \alpha) \in \hat{I} \times \bar{\Lambda}}$ is $q$-quantum. We set

$$
\delta_{\hat{I}, \bar{\Lambda}}^{(j, \beta)_{\mathrm{dc}}}=\operatorname{det}_{q}\left(x_{i, \alpha}^{(j, \beta)_{\mathrm{dc}}}\right)_{(i, \alpha) \in \hat{I} \times \bar{\Lambda}} .
$$

(4) Consider $\lambda^{\prime} \in \llbracket 1, p \rrbracket \backslash\left\{\alpha_{1}, \ldots, \alpha_{l}\right\}$. If $(j, \beta) \in E_{\mathrm{dc}}$ is greater (relative to $\leqslant_{\mathrm{dc}}$ ) than the elements of $\left\{i_{1}, \ldots, i_{l}\right\} \times\left\{\alpha_{1}, \ldots, \alpha_{k-1}, \alpha_{k+1}, \ldots, \alpha_{l}, \lambda^{\prime}\right\}$, it follows from Lemma 7.2 that the matrix

$$
\left(x_{i, \alpha}^{(j, \beta)_{\mathrm{dc}}}\right)_{(i, \alpha) \in\left\{i_{1}, \ldots, i_{l}\right\} \times\left\{\alpha_{1}, \ldots, \alpha_{k-1}, \alpha_{k+1}, \ldots, \alpha_{l}, \lambda^{\prime}\right\}}
$$

is $q$-quantum. We set

$$
\delta_{\alpha_{k} \rightarrow \lambda^{\prime}}^{(j, \beta)_{\mathrm{dc}}}=\operatorname{det}_{q}\left(x_{i, \alpha}^{(j, \beta)_{\mathrm{dc}}}\right)_{(i, \alpha) \in\left\{i_{1}, \ldots, i_{l}\right\} \times\left\{\alpha_{1}, \ldots, \alpha_{k-1}, \alpha_{k+1}, \ldots, \alpha_{l}, \lambda^{\prime}\right\}} .
$$

(5) Consider $i^{\prime} \in \llbracket 1, m \rrbracket \backslash\left\{i_{1}, \ldots, i_{l}\right\}$. If $(j, \beta) \in E_{\mathrm{dc}}$ is greater (relative to $\leqslant_{\mathrm{dc}}$ ) than the elements of $\left\{i_{1}, \ldots, i_{k-1}, i_{k+1}, \ldots, i_{l}, i^{\prime}\right\} \times\left\{\alpha_{1}, \ldots, \alpha_{l}\right\}$, it follows from Lemma 7.2 that the matrix

$$
\left(x_{i, \alpha}^{(j, \beta)_{\mathrm{dc}}}\right)_{(i, \alpha) \in\left\{i_{1}, \ldots, i_{k-1}, i_{k+1}, \ldots, i_{l}, i^{\prime}\right\} \times\left\{\alpha_{1}, \ldots, \alpha_{l}\right\}}
$$

is $q$-quantum. We set

$$
\delta_{i_{k} \rightarrow i^{\prime}}^{(j, \beta)_{\mathrm{dc}}}=\operatorname{det}_{q}\left(x_{i, \alpha}^{(j, \beta)_{\mathrm{dc}}}\right)_{(i, \alpha) \in\left\{i_{1}, \ldots, i_{k-1}, i_{k+1}, \ldots, i_{l}, i^{\prime}\right\} \times\left\{\alpha_{1}, \ldots, \alpha_{l}\right\}} .
$$

Using [10, Proposition 2.2.8], one can prove the following proposition.

Proposition 7.5. Let $M=\left(x_{i, \alpha}\right)_{(i, \alpha) \in[1, m \rrbracket \times \llbracket 1, p]}$ be a q-quantum matrix, let $(j, \beta) \in$ $E_{\mathrm{dc}}$ with $(j, \beta) \leqslant_{\mathrm{dc}}(m, p-1)$ and let

$$
\delta=\operatorname{det}_{q}\left(x_{i, \alpha}\right) \underset{\substack{i=i_{1}, \ldots, i_{l} \\ \alpha=\alpha_{1}, \ldots, \alpha_{l}}}{\alpha}
$$

be an $l \times l$ quantum minor of $M$ with $\left(i_{l}, \alpha_{l}\right)<_{\mathrm{dc}}(j, \beta)$. 
(1) If $t_{j, \beta}=0$, then $\delta^{(j, \beta)_{\mathrm{dc}}^{+}}=\delta^{(j, \beta)_{\mathrm{dc}}}$.

(2) Assume that $t_{j, \beta} \neq 0$.

If $i_{l}=j$, or if there exists $k \in \llbracket 1, l \rrbracket$ such that $\beta=\alpha_{k}$, or if $\beta<\alpha_{1}$, then $\delta^{(j, \beta)_{\mathrm{dc}}^{+}}=\delta^{(j, \beta)_{\mathrm{dc}}}$.

(3) Assume that $t_{j, \beta} \neq 0$ and that $i_{l}<j$.

(a) If $\alpha_{l}<\beta$, then

$$
\delta^{(j, \beta)_{\mathrm{dc}}^{+}}=\delta^{(j, \beta)_{\mathrm{dc}}}-\sum_{k=1}^{l}(-q)^{k-(l+1)} t_{j, \beta}^{-1} x_{j, \alpha_{k}}^{(j, \beta)_{\mathrm{dc}}} \delta_{\alpha_{k} \rightarrow \beta}^{(j, \beta)_{\mathrm{dc}}}
$$

and

$$
\delta^{(j, \beta)_{\mathrm{dc}}^{+}}=\delta^{(j, \beta)_{\mathrm{dc}}}-\sum_{k=1}^{l}(-q)^{(l+1)-k} \delta_{i_{k} \rightarrow j}^{(j, \beta)_{\mathrm{dc}}} x_{i_{k}, \beta}^{(j, \beta)_{\mathrm{dc}}} t_{j, \beta}^{-1} .
$$

(b) If there exists $h \in \llbracket 1, l-1 \rrbracket$ such that $\alpha_{h}<\beta<\alpha_{h+1}$, then

$$
\delta^{(j, \beta)_{\mathrm{dc}}^{+}}=\delta^{(j, \beta)_{\mathrm{dc}}}-\sum_{k=1}^{h}(-q)^{k-(h+1)} t_{j, \beta}^{-1} x_{j, \alpha_{k}}^{(j, \beta)_{\mathrm{dc}}} \delta_{\alpha_{k} \rightarrow \beta}^{(j, \beta)_{\mathrm{dc}}} .
$$

Proposition 7.6. Let $M=\left(x_{i, \alpha}\right)_{(i, \alpha) \in \llbracket 1, m \rrbracket \times \llbracket 1, p \rrbracket}$ be a q-quantum matrix, let $j \in$ $\llbracket 1, m \rrbracket$ and let

$$
\delta=\operatorname{det}_{q}\left(x_{i, \alpha}\right) \begin{gathered}
i=i_{1}, \ldots, i_{l} \\
\alpha=\alpha_{1}, \ldots, \alpha_{l}
\end{gathered}
$$

be an $l \times l$ quantum minor of $M$ with $\left(i_{l}, \alpha_{l}\right)<_{\mathrm{dc}}(j, p)$.

(1) If $t_{j, p}=0$, then $\delta^{(j, p)_{\mathrm{dc}}^{+}}=\delta^{(j, p)_{\mathrm{dc}}}$.

(2) Assume that $t_{j, p} \neq 0$.

If $\alpha_{l}=p$, or if there exists $k \in \llbracket 1, l \rrbracket$ such that $j=i_{k}$, or if $j<i_{1}$, then $\delta^{(j, p)_{\mathrm{dc}}^{+}}=\delta^{(j, p) \mathrm{dc}}$.

(3) Assume that $t_{j, p} \neq 0$ and that $\alpha_{l}<p$.

(a) If $i_{l}<j$, then

$$
\delta^{(j, p)_{\mathrm{dc}}^{+}}=\delta^{(j, p)_{\mathrm{dc}}}-\sum_{k=1}^{l}(-q)^{k-(l+1)} t_{j, p}^{-1} x_{i_{k}, p}^{(j, p)_{\mathrm{dc}}} \delta_{i_{k} \rightarrow j}^{(j, p) \mathrm{dc}}
$$

and

$$
\delta^{(j, p)_{\mathrm{dc}}^{+}}=\delta^{(j, p)_{\mathrm{dc}}}-\sum_{k=1}^{l}(-q)^{(l+1)-k} \delta_{\alpha_{k} \rightarrow p}^{(j, p)_{\mathrm{dc}}} x_{j, \alpha_{k}}^{(j, p)_{\mathrm{dc}}} t_{j, p}^{-1} .
$$


(b) If there exists $h \in \llbracket 1, l-1 \rrbracket$ such that $i_{h}<j<i_{h+1}$, then

$$
\delta^{(j, p)_{\mathrm{dc}}^{+}}=\delta^{(j, p)_{\mathrm{dc}}}-\sum_{k=1}^{h}(-q)^{k-(h+1)} t_{j, p}^{-1} x_{i_{k}, p}^{(j, p)_{\mathrm{dc}}} \delta_{i_{k} \rightarrow j}^{(j, p)_{\mathrm{dc}}} .
$$

Proof. We observe that the standard algorithm performed along the last row of a $q$-quantum matrix coincides with the last-column algorithm applied to the last column of its transpose. Since the algebras generated by a generic $q$-quantum matrix and by its transpose are isomorphic, this allows us to apply [10, Proposition 2.2.8] in order to obtain the result.

An immediate corollary of Propositions 7.5 and 7.6 is the following result.

Corollary 7.7. Let $M=\left(x_{i, \alpha}\right)_{(i, \alpha) \in \llbracket 1, m \rrbracket \times \llbracket 1, p \rrbracket}$ be a q-quantum matrix, let $(j, \beta) \in$ $E_{\mathrm{dc}} \backslash\{(m+1, p)\}$ and let

$$
\delta=\operatorname{det}_{q}\left(x_{i, \alpha}\right) \begin{gathered}
i=i_{1}, \ldots, i_{l} \\
\alpha=\alpha_{1}, \ldots, \alpha_{l}
\end{gathered}
$$

be an $l \times l$ quantum minor of $M$ with $\left(i_{l}, \alpha_{l}\right)<_{\mathrm{dc}}(j, \beta)$.

If either $i_{l}=j$ or $\alpha_{l}=\beta$, then $\delta^{(j, \beta)_{\mathrm{dc}}^{+}}=\delta^{(j, \beta)_{\mathrm{dc}}}$.

We finish this section by computing the quantum minors of $M^{(j, \beta)} \mathrm{dc}$ that involve $x_{j, \beta}^{(j, \beta)_{\mathrm{dc}}^{+}}$in terms of quantum minors of $M^{(j, \beta)_{\mathrm{dc}}}$.

Proposition 7.8. Let $M=\left(x_{i, \alpha}\right)_{(i, \alpha) \in \llbracket 1, m \rrbracket \times \llbracket 1, p \rrbracket}$ be a q-quantum matrix and let

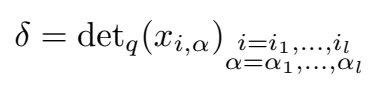

be an $l \times l$ quantum minor of $M$ with $l \geqslant 2$. Then

$$
\delta^{\left(i_{l}, \alpha_{l}\right)_{\mathrm{dc}}^{+}}=\delta_{\hat{i}_{l}, \bar{\alpha}_{l}}^{\left(i_{l}, \alpha_{l}\right)_{\mathrm{dc}}} t_{i_{l}, \alpha_{l}} .
$$

Proof. If $t_{i_{l}, \alpha_{l}}=x_{i_{l}, \alpha_{l}}^{\left(i_{l}, \alpha_{l}\right)_{\mathrm{dc}}^{+}}=0$, it follows from [3, Proposition 4.1.1] that $\delta^{\left(i_{l}, \alpha_{l}\right)_{\mathrm{dc}}^{+}}=0$. Thus,

$$
\delta^{\left(i_{l}, \alpha_{l}\right)_{\mathrm{dc}}^{+}}=0=\delta_{\hat{i}_{l}, \bar{\alpha}_{l}}^{\left(i_{l}, \alpha_{l}\right)_{\mathrm{dc}}} t_{i_{l}, \alpha_{l}} .
$$

Assume now that $t_{i_{l}, \alpha_{l}} \neq 0$ and set

$$
c_{i, \alpha}=x_{i, \alpha}^{\left(i_{l}, \alpha_{l}\right)_{\mathrm{dc}}^{+}} \text {for }(i, \alpha) \in\left\{i_{1}, \ldots, i_{l}\right\} \times\left\{\alpha_{1}, \ldots, \alpha_{l}\right\} .
$$

By Lemma 7.2, the matrix $C=\left(c_{i, \alpha}\right)_{(i, \alpha) \in\left\{i_{1}, \ldots, i_{l}\right\} \times\left\{\alpha_{1}, \ldots, \alpha_{l}\right\}}$ is $q$-quantum. Hence, we can apply the standard deleting-derivations algorithm to $C$ (see Conventions 3.3 ) and it is obvious that $c_{i, \alpha}^{(l, l)_{\mathrm{s}}}=x_{i, \alpha}^{\left(i_{l}, \alpha_{l}\right)_{\mathrm{dc}}}$ for all $(i, \alpha) \in\left\{i_{1}, \ldots, i_{l}\right\} \times\left\{\alpha_{1}, \ldots, \alpha_{l}\right\}$. So, we deduce from [3, Proposition 4.1.2] that

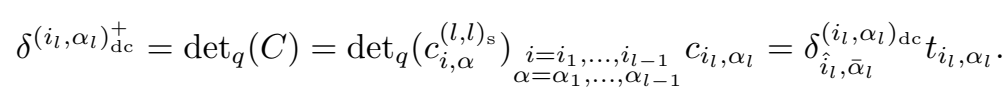




\section{Some vanishing criteria for quantum minors}

Throughout this section we use the following conventions.

(1) $K$ denotes a $\mathbb{C}$-algebra which is also a skew field.

(2) $m$ and $p$ are greater than or equal to 3 .

(3) $M=\left(x_{i, \alpha}\right)_{(i, \alpha) \in \llbracket 1, m \rrbracket \times \llbracket 1, p \rrbracket}$ is a $q$-quantum matrix with entries in $K$ and we set

$$
t_{i, \alpha}=x_{i, \alpha}^{(1,2)_{\mathrm{s}}}=x_{i, \alpha}^{(1,2)_{\mathrm{dc}}} \quad \text { for any }(i, \alpha) \in \llbracket 1, m \rrbracket \times \llbracket 1, p \rrbracket
$$

(see Proposition 6.3).

(4) We assume that the following property holds for the matrix $M$ : the non-zero monomials $t_{1,1}^{k_{1,1}} \cdots t_{m, p}^{k_{m, p}}$ (where the indices are increasing for $\leqslant_{\mathrm{s}}$ ) $\left(k_{i, \alpha} \in \mathbb{N}\right)$ are linearly independent over $\mathbb{C}$, so that $\mathbb{C}\left\langle t_{i, \alpha}\right|(i, \alpha) \in \llbracket 1, m \rrbracket \times \llbracket 1, p \rrbracket$ and $\left.t_{i, \alpha} \neq 0\right\rangle$ can be viewed as a quantum affine space.

\section{Notation 8.1.}

(i) $L$ denotes the matrix obtained from $M$ by deleting the last row and the last column, that is

$$
L=\left(x_{i, \alpha}\right)_{(i, \alpha) \in \llbracket 1, m-1 \rrbracket \times \llbracket 1, p-1 \rrbracket} .
$$

(ii) If $(j, \beta) \in E_{\mathrm{dc}}$, we denote by $L^{(j, \beta)_{\mathrm{dc}}}$ the matrix obtained from $M^{(j, \beta)_{\mathrm{dc}}}$ by deleting the last row and the last column, that is

$$
L^{(j, \beta)_{\mathrm{dc}}}=\left(x_{i, \alpha}^{(j, \beta)_{\mathrm{dc}}}\right)_{(i, \alpha) \in \llbracket 1, m-1 \rrbracket \times \llbracket 1, p-1 \rrbracket} .
$$

Observe that $L^{(m+1, p) \mathrm{dc}}=L$.

(iii) We set $N=L^{(m, 1)_{\mathrm{dc}}}$.

By Lemma 7.2, $N$ is a $q$-quantum matrix. Hence, the standard deleting-derivations algorithm (see Conventions 3.3) can be applied to $N$, and from Proposition 6.3 we deduce the following lemma.

Lemma 8.2. We have $N^{(1,2)_{\mathrm{s}}}=\left(t_{i, \alpha}\right)_{(i, \alpha) \in \llbracket 1, m-1] \times \llbracket 1, p-1 \rrbracket}$, so that the matrix $N$ satisfies convention (4) at the beginning of this section.

Lemma 8.3. Let $l \in \llbracket 1, \inf (m-1, p-1) \rrbracket$ and assume that all $l \times l$ quantum minors of $N$ are equal to 0 . If $(j, \beta) \in E_{\mathrm{dc}}$ with $(m, 1) \leqslant \mathrm{dc}(j, \beta) \leqslant \mathrm{dc}(m, p)$ and if $k$ is an integer such that $k \geqslant l$, then all $k \times k$ quantum minors of the q-quantum matrix $L^{(j, \beta) \mathrm{dc}}$ are equal to 0 .

Proof. If $k \geqslant l$, the $k \times k$ quantum minors of $L^{(j, \beta)_{\mathrm{dc}}}$ are right linear combinations (with coefficients in $K$ ) of $l \times l$ quantum minors of $L^{(j, \beta)_{\mathrm{dc}}}$. So, it is enough to prove that all $l \times l$ quantum minors of $L^{(j, \beta)_{\mathrm{dc}}}$ are zero. To achieve this aim, we proceed by iteration $($ for $\leqslant$ dc $)$ on $(j, \beta)$. 
Since $N=L^{(m, 1)_{\mathrm{dc}}}$, the case $(j, \beta)=(m, 1)$ is done. Assume now that $(m, 1) \leqslant \mathrm{dc}$ $(j, \beta) \leqslant \mathrm{dc}(m-1, p)$ and that all $l \times l$ quantum minors of $L^{(j, \beta)_{\mathrm{dc}}}$ are equal to 0 . In order to prove that the same property holds for all $l \times l$ quantum minors of $L^{(j, \beta)_{\mathrm{dc}}^{+}}$, two cases may be distinguished.

(i) If $j=m$, then $\beta<p$. Thus, by Proposition 7.5, every $l \times l$ quantum minor of $L^{(j, \beta)_{\mathrm{dc}}^{+}}$is a left linear combination (with coefficients in $K$ ) of $l \times l$ quantum minors of $L^{(j, \beta)_{\mathrm{dc}}}$. The desired result follows from the induction hypothesis.

(ii) If $j \neq m$, then $\beta=p$. Thus, by Proposition 7.6, every $l \times l$ quantum minor of $L^{(j, \beta)_{\mathrm{dc}}^{+}}$is a left linear combination (with coefficients in $K$ ) of $l \times l$ quantum minors of $L^{(j, \beta)_{\mathrm{dc}}}$. The desired result follows from the induction hypothesis.

Proposition 8.4. Let $l$ and $s$ be two integers such that $l \in \llbracket 1, \inf (m-1, p-1) \rrbracket$ and $s \in \llbracket 1, m-1 \rrbracket$, and assume that all $l \times l$ quantum minors of $N$ are equal to 0 . Then

(1) all $(l+1) \times(l+1)$ quantum minors of $M$ are equal to 0 ;

(2) if, moreover, we suppose that $x_{i, p}=t_{i, p}=0$ for $i \in \llbracket 1, s \rrbracket$, then all $l \times l$ quantum minors of the matrix obtained from $M$ by deleting the rows $s+1, \ldots, m$ are equal to 0 .

\section{Proof.}

(1) Let

$$
\delta=\operatorname{det}_{q}\left(x_{i, \alpha}\right) \underset{\substack{i=i_{1}, \ldots, i_{l+1} \\ \alpha=\alpha_{1}, \ldots, \alpha_{l+1}}}{\substack{\alpha_{1} \\ n_{1}}}
$$

be an $(l+1) \times(l+1)$ quantum minor of $M$. In order to establish that $\delta=0$, four cases are distinguished.

(i) If $\alpha_{l+1}=p$ and $i_{l+1}=m$, then, by Proposition 7.8, we have $\delta=\delta_{\hat{m}, \bar{p}}^{(m, p)_{\mathrm{dc}}} t_{m, p}$. Since $\delta_{\hat{m}, \bar{p}}^{(m, p)_{\mathrm{dc}}}$ is an $l \times l$ quantum minor of $L^{(m, p)_{\mathrm{dc}}}$, we deduce from Lemma 8.3 that

$$
\delta_{\hat{m}, \bar{p}}^{(m, p)_{\mathrm{dc}}}=0
$$

so that

$$
\delta=\delta_{\hat{m}, \bar{p}}^{(m, p) \mathrm{dc}} t_{m, p}=0 .
$$

(ii) If $\alpha_{l+1}=p$ and $i_{l+1}<m$, then, by Corollary 7.7 , we have $\delta=\delta^{\left(i_{l+1}+1, p\right)_{\mathrm{dc}}}$. Thus, it follows from Proposition 7.8 that

$$
\delta=\delta_{\hat{i}_{l+1}, \bar{p}}^{\left(i_{l+1}, p\right)_{\mathrm{dc}}} t_{i_{l+1}, p} .
$$

Since $\delta_{\hat{i}_{l+1}, \bar{p}}^{\left(i_{l+1}, p\right)_{\mathrm{dc}}}$ is an $l \times l$ quantum minor of $L^{\left(i_{l+1}, p\right)_{\mathrm{dc}}}$, we deduce from Lemma 8.3 that $\delta_{\hat{i}_{l+1}, \bar{p}}^{\left(i_{l+1}, p\right)_{\mathrm{dc}}}=0$. Hence

$$
\delta=\delta_{\hat{i}_{l+1}, \bar{p}}^{\left(i_{l+1}, p\right)_{\mathrm{dc}}} t_{i_{l+1}, p}=0
$$


(iii) If $\alpha_{l+1}<p$ and $i_{l+1}=m$, then, by Corollary 7.7, we have $\delta=\delta^{(m, p)_{\mathrm{dc}}}$. Expanding this last quantum minor along the last row (see [12, Corollary 4.4.4]), we get

$$
\delta=\sum_{k=1}^{l+1}(-q)^{k-(l+1)} x_{m, \alpha_{k}}^{(m, p)_{\mathrm{dc}}} \delta_{\hat{m}, \bar{\alpha}_{k}}^{(m, p)_{\mathrm{dc}}} .
$$

Since each $\delta_{\hat{m}, \bar{\alpha}_{k}}^{(m, p)}$ is an $l \times l$ quantum minor of $L^{(m, p)_{\mathrm{dc}}}$, we deduce from Lemma 8.3 that each $\delta_{\hat{m}, \bar{\alpha}_{k}}^{(m, p)_{\mathrm{dc}}}$ is equal to 0 , so that $\delta=0$.

(iv) If $\alpha_{l+1}<p$ and $i_{l+1}<m$, we have the following.

(a) If $t_{m, p}=0$, then it follows from Proposition 7.6 that $\delta=\delta^{(m, p))_{\mathrm{dc}}}$. Since $\delta^{(m, p)}$ dc is an $(l+1) \times(l+1)$ quantum minor of $L^{(m, p)}$ dc , we deduce from Lemma 8.3 that $\delta^{(m, p) \mathrm{dc}}=0$.

(b) Assume now that $t_{m, p} \neq 0$. By Proposition 7.6, we have

$$
t_{m, p} \delta=t_{m, p} \delta^{(m, p)_{\mathrm{dc}}}-\sum_{k=1}^{l+1}(-q)^{k-(l+2)} x_{i_{k}, p}^{(m, p)_{\mathrm{dc}}} \delta_{i_{k} \rightarrow m}^{(m, p)_{\mathrm{dc}}} .
$$

Since $\delta^{(m, p)_{\mathrm{dc}}}$ is an $(l+1) \times(l+1)$ quantum minor of $L^{(m, p))_{\mathrm{dc}}}$, we deduce from Lemma 8.3 that $\delta^{(m, p) \mathrm{dc}}=0$. Next, let $k \in \llbracket 1, l+1 \rrbracket$. Expanding $\delta_{i_{k} \rightarrow m}^{(m, p)}$ dc along the last row (see [12, Corollary 4.4.4]), we get

$$
\delta_{i_{k} \rightarrow m}^{(m, p)_{\mathrm{dc}}}=\sum_{i=1}^{l+1}(-q)^{i-(l+1)} x_{m, \alpha_{i}}^{(m, p)_{\mathrm{dc}}} \delta_{\hat{i}_{k}, \bar{\alpha}_{i}}^{(m, p)_{\mathrm{dc}}} .
$$

Since each $\delta_{\hat{i}_{k}, \bar{\alpha}_{i}}^{(m, p)_{\mathrm{dc}}}$ is an $l \times l$ quantum minor of $L^{(m, p)_{\mathrm{dc}}}$, we deduce from Lemma 8.3 that each $\delta_{\hat{i}_{k}, \bar{\alpha}_{i}}^{(m, p)}$ is equal to 0 . Thus $\delta_{i_{k} \rightarrow m}^{(m, p)_{\mathrm{dc}}}=0$.

Equation (8.1) and the above results show that $t_{m, p} \delta=0$, so that $\delta=0$. The proof of the first assertion is now complete.

(2) Let

$$
\delta=\operatorname{det}_{q}\left(x_{i, \alpha}\right) \underset{\substack{i=i_{1}, \ldots, i_{l} \\ \alpha=\alpha_{1}, \ldots, \alpha_{l}}}{\alpha}
$$

be an $l \times l$ quantum minor of $M$ with $i_{l} \leqslant s$. In order to show that $\delta=0$, two cases may be distinguished.

(i) Assume that $\alpha_{l}=p$. Thus, the last column of $\delta$ is 0 , so that $\delta=0$.

(ii) Assume that $\alpha_{l}<p$.

(a) If $t_{m, p}=0$, then $\delta=\delta^{(m, p)_{\mathrm{dc}}}$. Since $i_{l} \leqslant s<m$ and $\alpha_{l}<p, \delta^{(m, p)_{\mathrm{dc}}}$ is an $l \times l$ quantum minor of $L^{(m, p) \mathrm{dc}}$. Thus, it follows from Lemma 8.3 that $\delta^{(m, p) \mathrm{dc}}=0$. Hence $\delta=\delta^{(m, p))_{\mathrm{dc}}}=0$. 
(b) Assume now that $t_{m, p} \neq 0$. Since $i_{l} \leqslant s<m$ and $\alpha_{l}<p$, it follows from Proposition 7.6 that

$$
\delta t_{m, p}=\delta^{(m, p)_{\mathrm{dc}}} t_{m, p}-\sum_{k=1}^{l}(-q)^{l+1-k} \delta_{\alpha_{k} \rightarrow p}^{(m, p) \mathrm{dc}} x_{m, \alpha_{k}}^{(m, p) \mathrm{dc}} .
$$

Since $\delta^{(m, p) \mathrm{dc}}$ is an $l \times l$ quantum minor of $L^{(m, p) \mathrm{dc}}$, we deduce from Lemma 8.3 that $\left.\delta^{(m, p)}\right)_{\mathrm{dc}}=0$. Next, let $k \in \llbracket 1, l \rrbracket$. By Lemma 6.2 , we have $x_{i, p}^{(m, p))_{\mathrm{dc}}}=x_{i, p}$ for $i \in \llbracket 1, m \rrbracket$. Thus, since $i_{l} \leqslant s$, the last column of $\delta_{\alpha_{k} \rightarrow p}^{(m, p) \mathrm{dc}}$ is 0 , so that $\delta_{\alpha_{k} \rightarrow p}^{(m, p) \mathrm{dc}}=0$.

Equation (8.2) and the above results show that $\delta t_{m, p}=0$, so that $\delta=0$. The proof of the second assertion is now complete.

\section{Some non-vanishing criteria for quantum minors}

Throughout this section, we assume that the four conventions of $\S 8$ are satisfied and we retain the notation of that section.

\subsection{A criterion for $1 \times 1$ quantum minors}

Let $(i, \alpha) \in \llbracket 1, m \rrbracket \times \llbracket 1, p \rrbracket$ and assume that $t_{i, p} \times t_{m, \alpha} \neq 0$.

(i) If $i=m$, it follows from Lemma 6.2 that $x_{i, \alpha}=x_{m, \alpha}=t_{m, \alpha} \neq 0$.

(ii) If $\alpha=p$, it follows again from Lemma 6.2 that $x_{i, \alpha}=x_{i, p}=t_{i, p} \neq 0$.

(iii) If $i<m$ and $\alpha<p$, we have $x_{m, p} x_{i, \alpha}-x_{i, \alpha} x_{m, p}=-\left(q-q^{-1}\right) x_{i, p} x_{m, \alpha}$. So we deduce from Lemma 6.2 that $x_{m, p} x_{i, \alpha}-x_{i, \alpha} x_{m, p}=-\left(q-q^{-1}\right) t_{i, p} t_{m, \alpha} \neq 0$. This implies that $x_{i, \alpha} \neq 0$.

So we can conclude with the following proposition.

Proposition 9.1. Let $(i, \alpha) \in \llbracket 1, m \rrbracket \times \llbracket 1, p \rrbracket$ and assume that $t_{i, p} \times t_{m, \alpha} \neq 0$. Then $x_{i, \alpha} \neq 0$.

Remark 9.2. The above result is still true if $m=2$ or $p=2$.

\subsection{A criterion for quantum minors of $L$}

Notation 9.3. Let $(j, \beta) \in E_{\mathrm{dc}}$.

(i) We denote by $B^{(j, \beta)_{\mathrm{dc}}}$ the subalgebra of $K$ generated by the $x_{i, \alpha}^{(j, \beta)_{\mathrm{dc}}}((i, \alpha) \in$ $\llbracket 1, m \rrbracket \times \llbracket 1, p \rrbracket)$, that is

$$
B^{(j, \beta)_{\mathrm{dc}}}=\mathbb{C}\left\langle x_{i, \alpha}^{(j, \beta)_{\mathrm{dc}}} \mid(i, \alpha) \in \llbracket 1, m \rrbracket \times \llbracket 1, p \rrbracket\right\rangle .
$$


(ii) We denote by $C^{(j, \beta)_{\mathrm{dc}}}$ the subalgebra of $B^{(j, \beta)_{\mathrm{dc}}}$ defined by

$$
C^{(j, \beta)_{\mathrm{dc}}}=\mathbb{C}\left\langle x_{i, \alpha}^{(j, \beta)_{\mathrm{dc}}} \mid(1,1) \leqslant \mathrm{dc}_{\mathrm{dc}}(i, \alpha)<_{\mathrm{dc}}(j, \beta)\right\rangle .
$$

The following result is proved in the same manner as [10, Corollaire 3.5.5].

Lemma 9.4. Let $(j, \beta) \in E_{\mathrm{dc}}$. If $t_{j, \beta}=x_{j, \beta}^{(j, \beta)_{\mathrm{dc}}} \neq 0$, then the monomials $t_{j, \beta}^{k}(k \in \mathbb{N})$ are linearly independent in $B^{(j, \beta)_{\mathrm{dc}}}$ viewed as a right (respectively, left) $C^{(j, \beta)_{\mathrm{dc}}}$-module.

Proof. First, an induction (with respect to $\leqslant$ dc) shows that, if $(j, \beta) \in E_{\mathrm{dc}}$ and $(i, \alpha) \in \llbracket 1, m \rrbracket \times \llbracket 1, p \rrbracket$, then $x_{i, \alpha}^{(j, \beta)_{\mathrm{dc}}}=t_{i, \alpha}+Q$, where $Q$ is a Laurent polynomial (with coefficients in $\mathbb{C})$ in the non-zero $t_{u, \lambda}$ with $(1,1) \leqslant_{\mathrm{dc}}(u, \lambda)<_{\mathrm{dc}}(j, \beta)$. Hence, $C^{(j, \beta)_{\mathrm{dc}}}$ is contained in $D=\mathbb{C}\left\langle t_{i, \alpha}^{ \pm 1}\right|(1,1) \leqslant_{\mathrm{dc}}(i, \alpha)<_{\mathrm{dc}}(j, \beta)$ and $\left.t_{i, \alpha} \neq 0\right\rangle$. So it is enough to prove that the monomials $t_{j, \beta}^{k}(k \in \mathbb{N})$ are linearly independent in $K$ viewed as a left (respectively, right) $D$-module. This follows immediately from convention (4) of $\S 8$.

Proposition 9.5. Let $(j, \beta) \in E_{\mathrm{dc}} \backslash\{(m+1, p)\}$ and let

$$
\delta=\operatorname{det}_{q}\left(x_{i, \alpha}\right) \begin{gathered}
i=i_{1}, \ldots, i_{l} \\
\alpha=\alpha_{1}, \ldots, \alpha_{l}
\end{gathered}
$$

be an $l \times l$ quantum minor of $M$. Assume that $\left(i_{l}, \alpha_{l}\right)<_{\mathrm{dc}}(j, \beta)$. If $\delta^{(j, \beta)_{\mathrm{dc}}^{+}}=0$, then $\delta^{(j, \beta)_{\mathrm{dc}}}=0$.

Proof. If $t_{j, \beta}=0$, we have $\delta^{(j, \beta)_{\mathrm{dc}}}=\delta^{(j, \beta)_{\mathrm{dc}}^{+}}=0$, as required. Assume now that $t_{j, \beta} \neq$ 0 . We distinguish two cases.

(i) If $\beta=p$, then, since $\delta^{(j, \beta)_{\mathrm{dc}}^{+}}=0$, it follows from Proposition 7.6 that $t_{j, \beta} \delta^{(j, \beta)_{\mathrm{dc}}} \in$ $C^{(j, \beta)_{\mathrm{dc}}}$. On the other hand, it is clear that $\delta^{(j, \beta)_{\mathrm{dc}}} \in C^{(j, \beta)_{\mathrm{dc}}}$. Thus, we deduce from Lemma 9.4 that $\delta^{(j, \beta)_{\mathrm{dc}}}=0$.

(ii) If $\beta<p$, then, since $\delta^{(j, \beta)_{\mathrm{dc}}^{+}}=0$, it follows from Proposition 7.5 that $t_{j, \beta} \delta^{(j, \beta)_{\mathrm{dc}}} \in$ $C^{(j, \beta)_{\mathrm{dc}}}$. On the other hand, it is clear that $\delta^{(j, \beta)_{\mathrm{dc}}} \in C^{(j, \beta)_{\mathrm{dc}}}$. Thus, we deduce from Lemma 9.4 that $\delta^{(j, \beta)_{\mathrm{dc}}}=0$. The proof is now complete.

The following non-vanishing criterion can be easily deduced from Proposition 9.5.

Proposition 9.6. Let

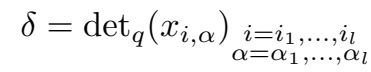

be an $l \times l$ quantum minor of $L$. If $\delta^{(m, 1)_{\mathrm{dc}}} \neq 0$, then $\delta \neq 0$. 


\subsection{A criterion for quantum minors of $M$}

Proposition 9.7. Let

$$
\delta=\operatorname{det}_{q}\left(x_{i, \alpha}\right) \underset{\substack{i=i_{1}, \ldots, i_{l} \\ \alpha=\alpha_{1}, \ldots, \alpha_{l}}}{ }
$$

be an $l \times l$ quantum minor of $M$ with $l \geqslant 2$, and assume that $t_{i_{l}, p} \times t_{m, \alpha_{l}} \neq 0$. If $\delta_{\hat{i}_{l}, \bar{\alpha}_{l}}^{(m, 1)} \neq 0$, then $\delta \neq 0$.

Proof. Assume that $\delta=0$. In order to prove that $\delta_{\hat{i}_{l}, \bar{\alpha}_{l}}^{(m, 1) \mathrm{dc}}=0$, three cases may be distinguished.

(i) If $\alpha_{l}=p$, then Corollary 7.7 shows that $\delta^{\left(i_{l}+1, p\right)_{\mathrm{dc}}}=\delta=0$. Since $t_{i_{l}, p} \neq 0$, it follows from Proposition 7.8 that $\delta_{\hat{i}_{l}, \bar{p}}^{\left(i_{l}, p\right)_{\mathrm{dc}}}=0$. Now, since $i_{l-1}<m$ and $\alpha_{l-1}<p$, we have $\left(i_{l-1}, \alpha_{l-1}\right)<_{\mathrm{dc}}(m, 1)$, and so, we deduce from Proposition 9.5 that $\delta_{\hat{i}_{l}, \bar{\alpha}_{l}}^{(m, 1) \mathrm{dc}}=0$, as desired.

(ii) If $i_{l}=m$ and $\alpha_{l}<p$, we deduce from Proposition 9.5 that $\delta^{\left(m, \alpha_{l}\right)_{\mathrm{dc}}^{+}}=0$. Thus, since $t_{m, \alpha_{l}} \neq 0$, Proposition 7.8 shows that $\delta_{\left.\hat{m}, \bar{\alpha}_{l}\right)}^{\left(m, \alpha_{l}\right)_{\mathrm{dc}}}=0$. Now, since $i_{l-1}<m$ and $\alpha_{l-1}<p$, we have $\left(i_{l-1}, \alpha_{l-1}\right)<_{\mathrm{dc}}(m, 1)$, and so, we deduce from Proposition 9.5 that $\delta_{\hat{i}_{l}, \bar{\alpha}_{l}}^{(m, 1)_{\mathrm{dc}}}=0$, as required.

(iii) If $i_{l}<m$ and $\alpha_{l}<p$, we observe that since $M$ is a $q$-quantum matrix, $x_{m, p} \neq 0$. Further, since $\alpha_{l}<p$, we have $\left(i_{l}, \alpha_{l}\right)<_{\mathrm{dc}}(m, p)$. It then follows from Proposition 9.5 that $\delta^{(m, p) \mathrm{dc}}=0$. Thus, by Lemma 6.2, formula (2) of [10, Proposition 2.2.8] gives us the equation

$$
0=\sum_{k=1}^{l}(-q)^{k-l-1} x_{m, \alpha_{k}} \delta_{\alpha_{k} \rightarrow p}^{(m, p))_{\mathrm{dc}}} .
$$

By Corollary 7.7, we have

$$
\delta_{\alpha_{k} \rightarrow p}^{(m, p)}=\delta_{\alpha_{k} \rightarrow p}^{\left(i_{l}+1, p\right)_{\mathrm{dc}}}
$$

Hence,

$$
\sum_{k=1}^{l}(-q)^{k-l-1} x_{m, \alpha_{k}} \delta_{\alpha_{k} \rightarrow p}^{\left(i_{l}+1, p\right) \mathrm{dc}}=0 .
$$

Now, we deduce from Proposition 7.8 that

$$
\sum_{k=1}^{l}(-q)^{k-l-1} x_{m, \alpha_{k}} \delta_{\hat{i}_{l}, \bar{\alpha}_{k}}^{\left(i_{l},\right)_{\mathrm{dc}}} t_{i_{l}, p}=0 .
$$

Since $t_{i_{l}, p} \neq 0$, we conclude that

$$
\sum_{k=1}^{l}(-q)^{k-l-1} x_{m, \alpha_{k}} \delta_{\hat{i}_{l}, \bar{\alpha}_{k}}^{\left(i_{l}, p\right)_{\mathrm{dc}}}=0 .
$$


On the other hand, by expanding

$$
\delta_{i_{l} \rightarrow m}^{\left(i_{l}, p\right)_{\mathrm{dc}}}=\operatorname{det}_{q}\left(\begin{array}{ccc}
x_{i_{1}, \alpha_{1}}^{\left(i_{l}, p\right)_{\mathrm{dc}}} & \cdots & x_{i_{1}, \alpha_{l}}^{\left(i_{l}, p\right)_{\mathrm{dc}}} \\
\vdots & \ddots & \vdots \\
x_{\left.i_{l}, p\right)_{\mathrm{dc}}}^{\left(i_{l}, \alpha_{1}\right.} & \cdots & x_{i_{l-1}, \alpha_{l}}^{\left(i_{l}, p\right)_{\mathrm{dc}}} \\
x_{m, \alpha_{1}}^{\left(i_{l},\right)_{\mathrm{dc}}} & \cdots & x_{m, \alpha_{l}}^{\left(i_{l}, p\right)_{\mathrm{dc}}}
\end{array}\right)
$$

along the last row (see [12, Corollary 4.4.4]), we obtain, by Lemma 6.2,

$$
\delta_{i_{l} \rightarrow m}^{\left(i_{l}, p\right)_{\mathrm{dc}}}=\sum_{k=1}^{l}(-q)^{k-l} x_{m, \alpha_{k}}^{\left(i_{l}, p\right)_{\mathrm{dc}}} \delta_{\hat{i}_{l}, \bar{\alpha}_{k}}^{\left(i_{l}, p\right)_{\mathrm{dc}}}=\sum_{k=1}^{l}(-q)^{k-l} x_{m, \alpha_{k}} \delta_{\hat{i}_{l}, \bar{\alpha}_{k}}^{\left(i_{l}, p\right)_{\mathrm{dc}}} .
$$

From (9.1), it follows that $\delta_{i_{l} \rightarrow m}^{\left(i_{l}, p\right)_{\mathrm{dc}}}=0$. Hence, by using Proposition 9.5, we get $\delta_{i_{l} \rightarrow m}^{\left(m, \alpha_{l}\right)_{\mathrm{dc}}^{+}}=0$. Thus, since $t_{m, \alpha_{l}} \neq 0$, it follows from Proposition 7.8 that $\delta_{\hat{i}_{l}, \bar{\alpha}_{l}}^{\left(m, \alpha_{l}\right)_{\mathrm{dc}}}=0$. Since $i_{l-1}<m$ and $\alpha_{l-1}<p$, we have $\left(i_{l-1}, \alpha_{l-1}\right)<_{\mathrm{dc}}(m, 1)$; so, Proposition 9.5 shows that $\delta_{\hat{i}_{l}, \bar{\alpha}_{l}}^{(m, 1)_{\mathrm{dc}}}=0$. The proof is now complete.

\section{A generating set for some $\mathcal{H}$-invariant prime ideals in $R$}

The aim of this last section is to construct a generating set of quantum minors for some $J_{w}$. To do this, we use the notation of $\S \S 2$ and 3 . Let $w \in W$. Recall that $J_{w}$ denotes the corresponding $\mathcal{H}$-invariant prime ideal in $R$ (see Proposition 2.8), that $F_{w}$ denotes the skew field of fractions of $R_{w}=R / J_{w}$, and that $M_{w}=\left(y_{i, \alpha}\right)_{(i, \alpha) \in \llbracket 1, m \rrbracket \times[1, p \rrbracket}$, where $y_{i, \alpha}=Y_{i, \alpha}+J_{w}$ (see Notation 3.6). If $(i, \alpha) \in \llbracket 1, m \rrbracket \times \llbracket 1, p \rrbracket$, we still set $t_{i, \alpha}=y_{i, \alpha}^{(1,2)_{\mathrm{s}}}$. We have shown (see Theorem 3.7) that

(i) $M_{w}$ is a $q$-quantum matrix with entries in $F_{w}$;

(ii) $t_{i, \alpha}=0$ if and only if $(i, \alpha) \in w$;

(iii) there exists an isomorphism from $\mathbb{C}\left\langle t_{i, \alpha} \mid(i, \alpha) \notin w\right\rangle$ onto the subalgebra $\mathbb{C}\left\langle T_{i, \alpha}\right|$ $(i, \alpha) \notin w\rangle$ of $\bar{R}_{\mathrm{s}}$ which sends $t_{i, \alpha}$ onto $T_{i, \alpha}$ for $(i, \alpha) \notin w$.

Thus, the conventions 1,3 and 4 of $\S \S 8$ and 9 are satisfied if we replace $K$ by $F_{w}, M$ by $M_{w}$ and $x_{i, \alpha}$ by $y_{i, \alpha}((i, \alpha) \in \llbracket 1, m \rrbracket \times \llbracket 1, p \rrbracket)$.

\subsection{The case $w=\llbracket 1, m-u \rrbracket \times \llbracket 1, p-u \rrbracket(u \geqslant 0)$}

By [10, Théorème 3.7.2], $J_{w}$ is generated by the quantum minors of $\mathcal{Y}$ which belong to $J_{w}$. So, in order to find a generating set for $J_{w}$, we just have to determine the quantum minors of $M_{w}$ which are equal to zero. To do this, we first establish the following result. 
Theorem 10.1. Let $K$ be a $\mathbb{C}$-algebra which is also a skew field, let $M=$ $\left(x_{i, \alpha}\right)_{(i, \alpha) \in \llbracket 1, m] \times \llbracket 1, p \rrbracket}$ be a $q$-quantum matrix with entries in $K$ and let $u \in \llbracket 0, \inf (m-1, p-$ $1) \rrbracket$. For $(i, \alpha) \in \llbracket 1, m \rrbracket \times \llbracket 1, p \rrbracket$, we set $t_{i, \alpha}=x_{i, \alpha}^{(1,2)_{s}}$. Assume that the non-zero monomials $t_{1,1}^{k_{1,1}} \cdots t_{m, p}^{k_{m, p}}$ (where the indices are increasing for $\leqslant_{\mathrm{s}}$ ) with $k_{i, \alpha} \in \mathbb{N}$ are linearly independent over $\mathbb{C}$. We also assume that $t_{i, \alpha}=0$ if and only if $(i, \alpha) \in \llbracket 1, m-u \rrbracket \times \llbracket 1, p-u \rrbracket$. Then

(1) the $v \times v$ quantum minors of $M$ with $v \geqslant u+1$ are zero;

(2) the $v \times v$ quantum minors of $M$ with $1 \leqslant v \leqslant u$ are non-zero.

Proof. If $u=0$, it is obvious that $x_{i, \alpha}=0$ for all $(i, \alpha) \in \llbracket 1, m \rrbracket \times \llbracket 1, p \rrbracket$, as required.

We now establish Theorem 10.1 when $m=2$ and $u=1$. In this case, it follows from Proposition 3.5 that we have

$$
x_{i, \alpha}= \begin{cases}t_{i, \alpha} & \text { if } i=2 \text { or } \alpha=p, \\ t_{i, p} t_{2, p}^{-1} t_{2, \alpha} & \text { otherwise. }\end{cases}
$$

Thus, all $1 \times 1$ quantum minors of $M$ are non-zero, as desired. Next, let

$$
\delta=\operatorname{det}_{q}\left(\begin{array}{ll}
x_{1, \alpha} & x_{1, \beta} \\
x_{2, \alpha} & x_{2, \beta}
\end{array}\right) \quad(1 \leqslant \alpha<\beta \leqslant p)
$$

be a $2 \times 2$ quantum minor of $M$. In order to show that $\delta=0$, we distinguish two cases.

(i) If $\beta<p$, then $\delta=t_{1, p} t_{2, p}^{-1} t_{2, \alpha} t_{2, \beta}-q t_{1, p} t_{2, p}^{-1} t_{2, \beta} t_{2, \alpha}$. Now, since $M^{(1,2)_{\mathrm{s}}}$ is a $(1,2)_{\mathrm{s}^{-}}$ $q$-quantum matrix, we have $t_{2, \beta} t_{2, \alpha}=q^{-1} t_{2, \alpha} t_{2, \beta}$, so that

$$
\delta=t_{1, p} t_{2, p}^{-1} t_{2, \alpha} t_{2, \beta}-t_{1, p} t_{2, p}^{-1} t_{2, \alpha} t_{2, \beta}=0 .
$$

(ii) If $\beta=p$, then $\delta=t_{1, p} t_{2, p}^{-1} t_{2, \alpha} t_{2, p}-q t_{1, p} t_{2, \alpha}$. Now, since $M^{(1,2)_{\mathrm{s}}}$ is a $(1,2)_{\mathrm{s}^{-}}$ $q$-quantum matrix, we have $t_{2, \alpha} t_{2, p}=q t_{2, p} t_{2, \alpha}$, so that

$$
\delta=q t_{1, p} t_{2, p}^{-1} t_{2, p} t_{2, \alpha}-q t_{1, p} t_{2, \alpha}=q t_{1, p} t_{2, \alpha}-q t_{1, p} t_{2, \alpha}=0 .
$$

Thus, all $2 \times 2$ quantum minors of $M$ are zero and Theorem 10.1 is now established when $m=2$ and $u=1$.

By a similar argument, we establish Theorem 10.1 when $p=2$ and $u=1$, and this proves Theorem 10.1 when $m=2$ or $p=2$.

We now assume that $m, p \geqslant 3$ and that the result is true for any $m^{\prime} \times p^{\prime} q$-quantum matrix with $\left(m^{\prime}, p^{\prime}\right)<_{s}(m, p)$. If $u=0$, we have already proved the desired result.

Assume now that $u \geqslant 1$. Since $m$ and $p$ are greater than or equal to 3 , the four conventions of $\delta \S 8$ and 9 are satisfied. Hence, we can use the notation and results of these two sections. In particular, we still denote by $N$ the matrix obtained from $M^{(m, 1)_{\mathrm{dc}}}$ by deleting the last row and the last column. By Lemma 8.2, the induction hypothesis can be applied to the $q$-quantum matrix $N$. This leads to the following properties. 
(1) The $v \times v$ quantum minors of $N$ with $v \geqslant u$ are equal to zero.

(2) The $v \times v$ quantum minors of $N$ with $1 \leqslant v \leqslant u-1$ are non-zero.

It then follows from Assertion 1 and Proposition 8.4 (with $l=v \geqslant u$ ) that the $(v+1) \times$ $(v+1)$ quantum minors of $M$ with $v \geqslant u$ are zero. On the other hand, since $t_{i, p} \times t_{m, \alpha} \neq 0$ for all $(i, \alpha) \in \llbracket 1, m \rrbracket \times \llbracket 1, p \rrbracket$, it follows from Proposition 9.1 that all $1 \times 1$ quantum minors of $M$ are non-zero, and it follows from Assertion 2 and Proposition 9.7 (with $l=v+1$ when $1 \leqslant v \leqslant u-1)$ that the $(v+1) \times(v+1)$ quantum minors of $M$ with $1 \leqslant v \leqslant u-1$ are non-zero. All this together shows that the $v \times v$ quantum minors of $M$ with $v \geqslant u+1$ are equal to zero and that the $v \times v$ quantum minors of $M$ with $1 \leqslant v \leqslant u$ are non-zero. This completes the inductive step and the result follows.

Let $u \in \llbracket 0, \inf (m-1, p-1) \rrbracket$ and set $w=\llbracket 1, m-u \rrbracket \times \llbracket 1, p-u \rrbracket$. Then $w$ is an element of $W$ and the matrix $M_{w}$ satisfies the hypotheses of Theorem 10.1. Since the $v \times v$ quantum minors with $v \geqslant u+1$ are right linear combinations (with coefficients in $R$ ) of $(u+1) \times(u+1)$ quantum minors, the following theorem results from Theorem 10.1 (and [10, Théorème 3.7.2]).

Theorem 10.2. Let $u$ be an integer such that $0 \leqslant u \leqslant \inf (m-1, p-1)$, and set $w=\llbracket 1, m-u \rrbracket \times \llbracket 1, p-u \rrbracket$. Then $w$ belongs to $W$ and $J_{w}$ is generated by the $(u+1) \times(u+1)$ quantum minors of $\mathcal{Y}$.

Remark 10.3. Let $u$ be an integer such that $0 \leqslant u \leqslant \inf (m-1, p-1)$. It follows from Theorem 10.2 that the ideal generated by the $(u+1) \times(u+1)$ quantum minors of $\mathcal{Y}$ is (completely) prime. So, we have just established that the quantum determinantal ideals are (completely) prime. In the more general case where we only assume that $q$ is a non-zero element of any base field, this result was proved by Goodearl and Lenagan (see $[\mathbf{4}$, Corollary 2.6]) by using different methods.

10.2. The case $w=(\llbracket 1, m-u \rrbracket \times \llbracket 1, p-u \rrbracket) \cup(\llbracket 1, s \rrbracket \times\{p\})(u \geqslant 1, s \geqslant 1)$

Theorem 10.4. Assume that $m$ and $p$ are greater than or equal to 3 , and let $u$ and $s$ be two integers such that $u \in \llbracket 1, \inf (m-1, p-1) \rrbracket$ and $1 \leqslant s \leqslant m-1$. Set $w=(\llbracket 1, m-u \rrbracket \times \llbracket 1, p-u \rrbracket) \cup(\llbracket 1, s \rrbracket \times\{p\})$. Then $w$ belongs to $W$ and $J_{w}$ is generated by

(1) the $(u+1) \times(u+1)$ quantum minors of $\mathcal{Y}$;

(2) the $u \times u$ quantum minors of the matrix obtained from $\mathcal{Y}$ by deleting the rows $s+1, \ldots, m$;

(3) $Y_{1, p}, \ldots, Y_{s, p}$.

Proof. By [10, Théorème 3.7.2], $J_{w}$ is generated by the quantum minors of $\mathcal{Y}$ which belong to $J_{w}$. So, in order to find a generating set for $J_{w}$, we just have to find the quantum minors of $M_{w}$ which are equal to 0 . This is what we do now. 
Since $m$ and $p$ are greater than or equal to 3 , the four conventions of $\S \S 8$ and 9 are satisfied if we replace $K$ by $F_{w}, M$ by $M_{w}$ and $x_{i, \alpha}$ by $y_{i, \alpha}((i, \alpha) \in \llbracket 1, m \rrbracket \times \llbracket 1, p \rrbracket)$. So, we can use the notation and results of these two sections. In particular, $N$ still denotes the matrix obtained from $M_{w}^{(m, 1)_{\mathrm{dc}}}$ by deleting the last row and the last column.

Now if $(i, \alpha) \in \llbracket 1, m-1 \rrbracket \times \llbracket 1, p-1 \rrbracket$, it follows from Theorem 3.7 that $t_{i, \alpha}=0$ if and only if $(i, \alpha) \in \llbracket 1, m-u \rrbracket \times \llbracket 1, p-u \rrbracket$. Hence, it follows from Lemma 8.2 that $N$ satisfies the hypotheses of Theorem 10.1 if we replace $m$ by $m-1, p$ by $p-1$ and $u$ by $u-1$. Thus, the $v \times v$ quantum minors of $N$ with $v \geqslant u$ are zero and the $v \times v$ quantum minors of $N$ with $v \leqslant u-1$ are non-zero. It then follows from the first assertion of Proposition 8.4 (with $l=v \geqslant u$ ) that the $(v+1) \times(v+1)$ quantum minors of $M_{w}$ with $v \geqslant u$ are zero. Hence, the quantum minors

with $v \geqslant u+1$ belong to $J_{w}$.

$$
\operatorname{det}_{q}\left(Y_{i, \alpha}\right) \underset{\substack{i=i_{1}, \ldots, i_{v} \\ \alpha=\alpha_{1}, \ldots, \alpha_{v}}}{\alpha_{2}}
$$

It remains to deal with the $v \times v$ quantum minors of $M_{w}$ such that $1 \leqslant v \leqslant u$. Let

$$
\delta=\operatorname{det}_{q}\left(y_{i, \alpha}\right) \begin{gathered}
i=i_{1}, \ldots, i_{v} \\
\alpha=\alpha_{1}, \ldots, \alpha_{v}
\end{gathered}
$$

be such a quantum minor. We consider four cases.

(i) Assume that $v=u$ and that $i_{u} \leqslant s$. It follows from the second assertion of Proposition 8.4 (with $l=u$ ) that $\delta=0$. Hence, the quantum minors

$$
\operatorname{det}_{q}\left(Y_{i, \alpha}\right) \begin{gathered}
i=i_{1}, \ldots, i_{u} \\
\alpha=\alpha_{1}, \ldots, \alpha_{u}
\end{gathered}
$$

with $i_{u} \leqslant s$ belong to $J_{w}$.

(ii) Assume that $1<v \leqslant u$ and that $i_{v}>s$. Recall that, if $1 \leqslant k \leqslant u-1$, the $k \times k$ quantum minors of $N$ are non-zero. In particular, $\delta_{\hat{i}_{v}, \bar{\alpha}_{v}}^{(m, 1)_{\mathrm{dc}}} \neq 0$. Thus, since $t_{i_{v}, p} \times t_{m, \alpha_{v}} \neq 0$ (remember that $i_{v}>s$ ), it follows from Proposition 9.7 that $\delta$ is non-zero. Hence, the quantum minors

$$
\operatorname{det}_{q}\left(Y_{i, \alpha}\right) \underset{\substack{i=i_{1}, \ldots, i_{v} \\ \alpha=\alpha_{1}, \ldots, \alpha_{v}}}{\alpha_{2}}
$$

with $1<v \leqslant u$ and $i_{v}>s$ do not belong to $J_{w}$.

(iii) Assume that $1 \leqslant v<u$ and that $i_{v} \leqslant s$. If $\alpha_{v}=p$, then the last column of $\delta$ is zero, so that $\delta=0$. Hence, the quantum minors

$$
\operatorname{det}_{q}\left(Y_{i, \alpha}\right) \begin{gathered}
i=i_{1}, \ldots, i_{v} \\
\alpha=\alpha_{1}, \ldots, \alpha_{v}
\end{gathered}
$$

with $1 \leqslant v<u, i_{v} \leqslant s$ and $\alpha_{v}=p$ belong to $J_{w}$.

If $\alpha_{v}<p$, then, since $i_{v} \leqslant s<m, \delta^{(m, 1) \mathrm{dc}}$ is a $v \times v$ quantum minor of $N$. Thus, since $v<u$, we have $\delta^{(m, 1) \mathrm{dc}} \neq 0$. It then follows from Proposition 9.6 that $\delta \neq 0$. Hence, the quantum minors

$$
\operatorname{det}_{q}\left(Y_{i, \alpha}\right) \begin{gathered}
i=i_{1}, \ldots, i_{v} \\
\alpha=\alpha_{1}, \ldots, \alpha_{v}
\end{gathered}
$$

with $1 \leqslant v<u, i_{v} \leqslant s$ and $\alpha_{v}<p$ do not belong to $J_{w}$. 
(iv) Assume that $v=1$ and that $i_{v}>s$. Observe that $t_{i_{v}, p} \times t_{m, \alpha_{v}} \neq 0$ (since $i_{v}>s$ ). Thus, it follows from Proposition 9.1 that $\delta=y_{i_{v}, \alpha_{v}} \neq 0$. Hence, the quantum minors

$$
\operatorname{det}_{q}\left(Y_{i, \alpha}\right) \begin{gathered}
i=i_{1}, \ldots, i_{v} \\
\alpha=\alpha_{1}, \ldots, \alpha_{v}
\end{gathered}
$$

with $v=1$ and $i_{v}>s$ do not belong to $J_{w}$.

We deduce from the above results that $J_{w}$ is generated by

(i) the $v \times v$ quantum minors with $v \geqslant u+1$;

(ii) the $u \times u$ quantum minors of the matrix obtained from $\mathcal{Y}$ by deleting the rows $s+1, \ldots, m$;

(iii) the $v \times v$ quantum minors with $1 \leqslant v<u, \alpha_{v}=p$ and $i_{v} \leqslant s$.

Denote by $L_{w}$ the two-sided ideal in $R$ generated by the $(u+1) \times(u+1)$ quantum minors of $\mathcal{Y}$, by the $u \times u$ quantum minors of the matrix obtained from $\mathcal{Y}$ by deleting the rows $s+1, \ldots, m$ and by $Y_{1, p}, \ldots, Y_{s, p}$. The above results show that $L_{w} \subseteq J_{w}$. Since the $v \times v$ quantum minors with $v \geqslant u+1$ are left linear combinations (with coefficients in $R$ ) of $(u+1) \times(u+1)$ quantum minors, and since the $v \times v$ quantum minors with $1 \leqslant v<u$, $\alpha_{v}=p$ and $i_{v} \leqslant s$ are left linear combinations of $Y_{1, p}, \ldots, Y_{s, p}$, we have $J_{w} \subseteq L_{w}$. Hence $J_{w}=L_{w}$ and the proof is complete.

\subsection{The case $w=(\llbracket 1, m-u \rrbracket \times \llbracket 1, p-u \rrbracket) \cup(\llbracket 1, s \rrbracket \times\{p\})(u>s \geqslant 1)$}

An immediate corollary of Theorem 10.4 is the following result.

Corollary 10.5. Assume that $m$ and $p$ are greater than or equal to 3 and let $u$ and $s$ be two integers such that $1 \leqslant s<u \leqslant \inf (m-1, p-1)$. Set $w=(\llbracket 1, m-u \rrbracket \times \llbracket 1, p-$ $u \rrbracket) \cup(\llbracket 1, s \rrbracket \times\{p\})$. Then $w$ belongs to $W$ and $J_{w}$ is generated by

(1) the $(u+1) \times(u+1)$ quantum minors of $\mathcal{Y}$;

(2) $Y_{1, p}, \ldots, Y_{s, p}$

The following result can be easily deduced from Corollary 10.5 (with $u \geqslant 2$ and $s=1$ ).

Corollary 10.6. Assume that $m$ and $p$ are greater than or equal to 3 and let $u \in$ $\llbracket 2, \inf (m-1, p-1) \rrbracket$. The two-sided ideal in $R$ generated by the $(u+1) \times(u+1)$ quantum minors of $\mathcal{Y}$ and by $Y_{1, p}$ is (completely) prime.

Remark 10.7. Corollary 10.6 allowed Lenagan and Rigal [11] to show that the quantum determinantal factor rings of $O_{q}\left(\mathcal{M}_{m, p}(\mathbb{C})\right)$ are maximal orders. 


\section{References}

1. G. Cauchon, Quotients premiers de $O_{q}\left(\mathcal{M}_{n}(k)\right)$, J. Alg. 180 (1996), 530-545.

2. G. CAUCHON, Effacement des dérivations et spectres premiers des algèbres quantiques, $J$. Alg. 260 (2003), 476-518.

3. G. CAUCHON, Spectre premier de $O_{q}\left(\mathcal{M}_{n}(k)\right)$, image canonique et séparation normale, J. Alg. 260 (2003), 519-569.

4. K. R. Goodearl and T. H. Lenagan, Quantum determinantal ideals, Duke Math. J. 103 (2000), 165-190.

5. K. R. Goodearl and T. H. Lenagan, Prime ideals invariant under winding automorphisms in quantum matrices, Int. J. Math. 13 (2002), 497-532.

6. K. R. Goodearl and T. H. Lenagan, Winding-invariant prime ideals in quantum $3 \times 3$ matrices, J. Alg. 260 (2003), 657-687.

7. K. R. Goodearl and E. S. Letzter, Prime factor algebras of the coordinate ring of quantum matrices, Proc. Am. Math. Soc. 121 (1994), 1017-1025.

8. K. R. Goodearl and E. S. Letzter, The Dixmier-Moeglin equivalence in quantum matrices and quantum Weyl algebras, Trans. Am. Math. Soc. 352 (2000), 1381-1403.

9. K. R. Goodearl And E. S. Letzter, Prime and primitive spectra of multiparameter quantum affine spaces, in Trends in Ring Theory, Miskolc, 1996, CMS Conf. Proc., vol. 22, pp. 39-58 (American Mathematical Society, Providence, RI, 1998).

10. S. Launois, Les idéaux premiers invariants de $O_{q}\left(\mathcal{M}_{m, p}(\mathbb{C})\right), J$. Alg., in press.

11. T. H. LENAGAN AND L. RigaL, The maximal order property for quantum determinantal rings, Proc. Edinb. Math. Soc. 46 (2003), 513-529.

12. B. J. PARshall And J. P. WANG, Quantum linear groups, Memoirs of the American Mathematical Society, vol. 439 (1991). 